\title{
Real-Time Illumination and Visual Coherence for Photorealistic Augmented/Mixed Reality
}

A'AESHAH ALHAKAMY, Indiana University-Purdue University Indianapolis, USA and University of Tabuk, Saudi Arabia

MIHRAN TUCERYAN, Indiana University-Purdue University Indianapolis, USA

A realistically inserted virtual object in the real-time physical environment is a desirable feature in augmented reality (AR) applications and mixed reality (MR) in general. This problem is considered a vital research area in computer graphics, a field that is experiencing ongoing discovery. The algorithms and methods used to obtain dynamic and real-time illumination measurement, estimating, and rendering of augmented reality scenes are utilized in many applications to achieve a realistic perception by humans. We cannot deny the powerful impact of the continuous development of computer vision and machine learning techniques accompanied by the original computer graphics and image processing methods to provide a significant range of novel AR/MR techniques. These techniques include methods for light source acquisition through image-based lighting or sampling, registering and estimating the lighting conditions, and composition of global illumination. In this review, we discussed the pipeline stages with the details elaborated about the methods and techniques which contributed to the development of providing a photo-realistic rendering, visual coherence, and interactive real-time illumination results in AR/MR.

CCS Concepts: • Computing methodologies $\rightarrow$ Computer graphics; Graphics systems and interfaces; Mixed/augmented reality; Perception.

Additional Key Words and Phrases: Augmented Reality, mixed reality, Visual Coherence, Real-Time, Illumination, lighting condition, Image-Based Lighting, Reflectance, and Shading, Photo-realistic.

ACM Reference Format:

A'aeshah Alhakamy and Mihran Tuceryan. 2020. Real-Time Illumination and Visual Coherence for Photorealistic Augmented/Mixed Reality. ACM Comput. Surv. 1, 1, Article 1 (January 2020), 35 pages. https://doi.org/10.1145/3386496

\section{INTRODUCTION}

As is known, virtual reality (VR) provides a totally computer-generated environment for interaction with the user, while augmented reality (AR) embeds virtual contents that are registered directly to the physical environment. Augmented reality links the gap between the virtual and real worlds, in both spatial and cognitive substance. In AR applications, the user's perception of the digital information is integrated and perceived as part of the real world. Furthermore, the Mixed Reality (MR) also refers to a hybrid reality for producing an innovative visualization and environments where the digital and physical objects interact and co-exist in real-time.

Authors' addresses: A'aeshah Alhakamy, aalhakam@iu.edu, aalhakami@ut.edu.sa, Indiana University-Purdue University Indianapolis, Indiana, USA, University of Tabuk, Saudi Arabia; Mihran Tuceryan, tuceryan@iu.edu, Indiana University-Purdue University Indianapolis, Indiana, USA.

Permission to make digital or hard copies of all or part of this work for personal or classroom use is granted without fee provided that copies are not made or distributed for profit or commercial advantage and that copies bear this notice and the full citation on the first page. Copyrights for components of this work owned by others than ACM must be honored. Abstracting with credit is permitted. To copy otherwise, or republish, to post on servers or to redistribute to lists, requires prior specific permission and/or a fee. Request permissions from permissions@acm.org.

(c) 2020 Association for Computing Machinery.

Manuscript submitted to ACM

Manuscript submitted to ACM

This is the author's manuscript of the article published in final edited form as:

Alhakamy, A., \& Tuceryan, M. (2020). Real-time Illumination and Visual Coherence for Photorealistic Augmented/Mixed Reality. ACM Computing Surveys, 53(3), 49:1-49:34. https://doi.org/10.1145/3386496 


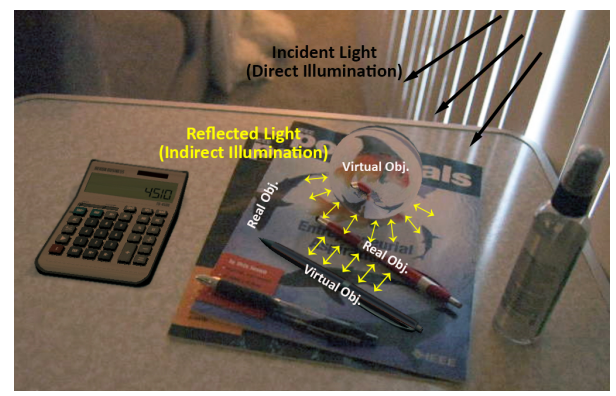

Fig. 1. Illustration of the light interaction between each type of objects; virtual and real $[6,7]$

Some of the previous work were defined without output devices specification such as head-mounted displays (HMD) [78], mobile devices, and cameras in order to limit the AR visual media. Thus, it may be difficult to realize that other other information were captured from the surrounding environment including audio, haptic, olfactory, or gustatory AR. It entails spatial registration and real-time control, which means precise alignment in 3D in real-time for corresponding real and virtual information. The user of the AR system under this mandate could use at least some viewpoint control interactively, and the display of computer-registered augmentations will remain registered in the environment for each referenced object.

Many AR/MR applications nowadays can produce fair results, but they still lack the realistic output in which the human eye could be easily deceived and not recognize the virtual objects from the real ones. The ability to synthesize realistic images and integrate virtual objects flawlessly into physical environment scenes is one of the significant objectives of many AR systems. The ability to produce a photo-realistic augmented object depicting the real world illumination characteristic such as incident light, reflection, shading, and cast shadows, is becoming increasingly important.

Therefore, a large number of techniques were developed for capturing and estimating the light source and its conditions in the physical world. Also, many techniques were developed to augment objects in the right position. Computer vision, machine learning, and high dynamic range imaging (HDR) techniques expedited a variety of novel estimating and rendering methods. This paper aims to provide a comprehensive review and comparison of capturing and estimating illumination techniques for augmented reality. For further reading, the reader is referred to the book by Schmalstieg and Hoellerer [95] where most of the concepts and methods mentioned in this paper are described in detail.

Accurate registration and calibration for a consistent geometry was the focal point in the early research work, but which omits the effects of local or global illumination and cast shadows among virtual and real objects. Ignoring the impact of illumination effects during the augmentation of the scene resulted in producing poor-quality and sometimes visually confusing outputs. The current studies have shown that one of the critical elements for virtual objects to be perceived as realistic objects in real scenes is their photo-realistic rendering with an illumination consistent with the physical scene [12, 36, 48, 89, 95].

The idea is not only to illuminate the virtual objects with captured or estimated incident light in the real environment, but to further simulate cast shadows and reflected light interactions as a unified illumination among the different types of objects in the scene, whether they are virtual or real, see Figure 1. In this review, we provide a survey about multiple methods that accomplished illumination effects of a dynamic scene in different stages for more visual coherence in real-time.

Manuscript submitted to ACM 


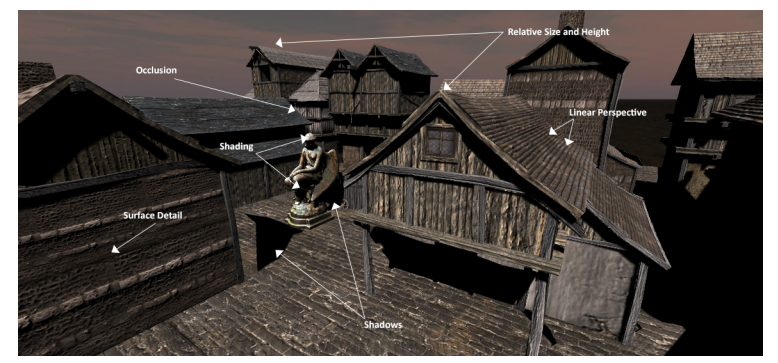

Fig. 2. Monocular depth cues allow scene structure interpretation from a single image.

We review methods on the sequence of familiar stages of mixed reality for acquisition, registration (estimation), composition and display to obtain a consistent illumination that includes the following points:

- Capturing the real world light source using typical measurements using, for instance, omnidirectional HDR images.

- Estimating the light environment from images and video directly.

- Compositing the dynamic radiometric information from virtual to a real object such as shadow casting.

- Rendering efficient techniques in augmented reality using Monte Carlo ray tracing, precomputed radiance transport, and differential rendering.

For a more organized and focused survey, we are omitting the geometric calibration and registration aspects of this topic. Therefore, we only cover methods that involve illumination and lighting. However, the reader is referred to the book [95] for more details. We include recent advances in studies and research from computer vision, computer graphics and virtual/augmented reality. Before we dive into those specific details, we need to explore a small overview of the visual coherence, rendering equation, and light transfer model.

\section{VISUAL COHERENCE: AN OVERVIEW}

The ability to embed a three-dimensional virtual object into an image of the real scene means that objects should be rendered from a virtual camera with internal and external parameters which correspond to the physical camera viewing the physical scene. Fundamental depth cues can be obtained with such a calibrated camera. Information about the depth provides interpretation of three-dimensional structure from the viewpoint of the camera. Depth cues can be categorized as monocular or binocular where there are around 15-20 different depth cues. Cues from a single image are known as monocular, see Figure 2, while cues depicted in a pair of images are called binocular [41, 55, 95].

At present, several AR displays use a monocular video see-through mode. In general, for AR, the most important cue is the depth which can be produced by computer graphics software. Also, there are other essential depth cues such as:

- Relative size: the distance between the objects and the observer. The further it is, the smaller the object appears.

- Relative height: how far the object is from the other objects and where their base is higher in the image.

- Perspective: the convergence of the parallel lines as the distance from the observer increases.

- Surface detail: objects closer to the observer have more texture gradient and more fine-grained surface detail.

- Atmospheric attenuation: while closer objects appear clearer, most-distant objects can be blurred due to atmospheric effects.

- Occlusion: in the screen space, closer objects obscure the further ones along the line of sight. 
- Shading: according to the source of light, the objects are illuminated resulting in the shading of the surfaces due to geometry.

- Shadow: objects blocking the light cast shadow on other objects.

These cues are delivered by the well-equipped three-dimensional computer graphics tools. Some of them are more straightforward to produce by a virtual camera registered geometrically with a real one, such as size, perspective, height, and surface details. Atmospheric attenuation concerns far-field outdoor AR. However, the other cues, especially occlusion, shading, and shadows demand attention in the AR rendering process [41, 55, 95].

Combining the real and virtual worlds in $\mathrm{AR} / \mathrm{MR}$ extends the conventional rendering process in computer graphics pipeline to involve more steps. The video see-through pipeline is better suited in this paper than an optical see-through pipeline, which consists of the following stages:

- Acquisition: obtain a model or a set of data from the real scene such as geometry, materials, and illumination.

- Registration: transform the obtained sets of data which is the standard photometric and geometric properties of one coordinate system of the real and virtual scenes.

- Compositing: merge the virtual objects and the real physical environment into one single image scene.

- Display: provide the user with the composited image.

The rendering process in AR/MR obviously is more complicated than the standard pipeline in computer graphics. In $\mathrm{AR}$, we have to deal with the virtual scene and the real scene simultaneously providing geometric and photometric characteristics for both scenes [95]. In this report, we discuss each stage with elaborated details about the methods and technologies which contributed to the development of providing a photo-realistic rendering and dynamic real-time illumination results in $\mathrm{AR} / \mathrm{MR}$.

\section{RENDERING EQUATIONS AND LIGHT MODELS}

Writing code about a three-dimensional rendering engine acquaints one with many lighting models, and general concepts and definitions such as albedo, ambient lights, specular reflections, and diffuse colors. The reflection model of the diffuse surface (Lambertian surface) is the most straightforward lighting model also known as "dot product lighting" or "cosine rule." The intensity of each light source also known as RGB color reflected from a surface is given by the following equation:

$$
I=s C \times \sum_{i=1}^{\text {nlights }} l I_{i} \times\left(N \cdot L_{i}\right)
$$

Where $s C$ is the color of the surface and $l I$ represents the color of the light among multiple number of lights. The first fragment of the code is to calculate the incoming light from different directions, scale it by the cosine of the angle between surface normal $(N)$ and light source direction $(L)$. Then, multiply the outcome by the reflection function for the diffuse surface which is a constant color [31].

This is the purest form of the rendering equation based on the physics in order to produce images in computer graphics. It is a standard concept where the entire realistic lighting must be measured. The rendering equation [46] in Figure 3 is represented as follows:

$$
L_{o}\left(p, \vec{w}_{o}\right)=L_{e}\left(p, \vec{w}_{o}\right)+\int_{S} f_{r}\left(p, \vec{w}_{i}, \vec{w}_{o}\right) L\left(p^{\prime}, \vec{w}_{i}\right) G\left(p, p^{\prime}\right) V\left(p, p^{\prime}\right) d w_{i}
$$

Manuscript submitted to ACM 


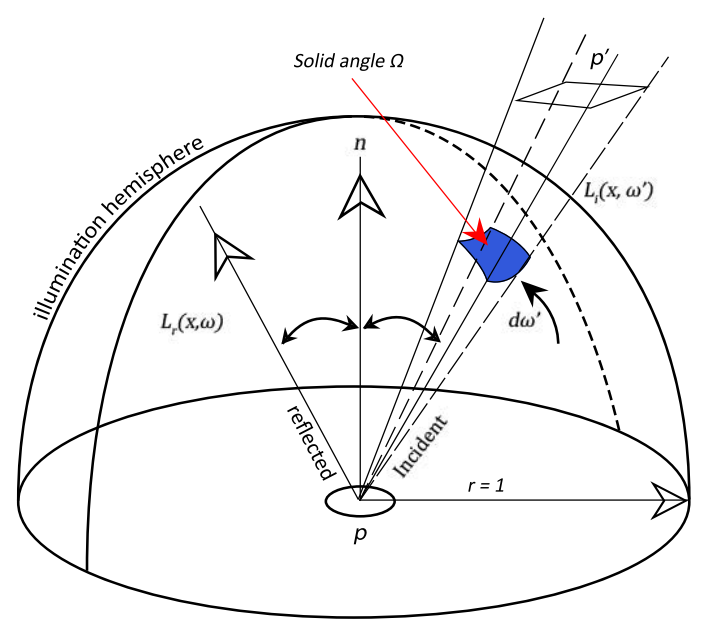

Fig. 3. Simple representation of the rendering equation.

Where $L_{o}\left(p, \vec{w}_{o}\right)$ represents the output radiance or the intensity reflected from position $\mathrm{p}$ into the reflection direction $\vec{w}_{o}$. The second part of equation $L_{e}\left(p, \vec{w}_{o}\right)$ refer to the emitted radiance/light from material at point $\mathrm{x}$ by the object itself. Then we have $S$ which describes the upper hemisphere surrounding the surface normal at the point $p$. The expression $f_{r}\left(p, \vec{w}_{i}, \vec{w}_{o}\right)$ represents the bidirectional reflectance distribution function of the surface (BRDF) at point $p$. Additionally, $L\left(p^{\prime}, \vec{w}_{i}\right)$ accounts for the incoming radiance at point $p^{\prime}$ arriving from all the directions $\vec{w}_{i}$ according to the BRDF and the surface normal. Also, $G\left(p, p^{\prime}\right)$ represents the geometric relationship between points $p$ and $p^{\prime}$, and $V\left(p, p^{\prime}\right)$ is a visibility test for diminished intensity per unit area which returns 1 if $p$ could see $p^{\prime}$ or returns 0 otherwise.

The only tricky concept while writing ray tracers is the usage of differential angles for representing a series of rays and the integral self-solving, where the background of global illumination is quite achievable [31]. Separating the incident radiance into different parts helps us comprehend the light transport in AR/MR where mixed scenes are involved:

$$
L\left(p^{\prime}, \vec{w}_{i}\right)=L^{r}\left(p^{\prime}, \vec{w}_{i}\right)+L^{v}\left(p^{\prime}, \vec{w}_{i}\right)+L^{v, r}\left(p^{\prime}, \vec{w}_{i}\right)
$$

The first part of the incidence radiance is $L^{r}\left(p^{\prime}, \vec{w}_{i}\right)$ which refers to the real scene incident radiance that is not yet reflected on the surface of the virtual objects. The second part, $L^{v}\left(p^{\prime}, \vec{w}_{i}\right)$ represents the incident radiance which could be emitted from either the virtual and real objects then reflected once or multiple times on the surfaces of the virtual objects. The third part, $L^{v, r}\left(p^{\prime}, \vec{w}_{i}\right)$ stands for the incident radiance which already interacts with both real and virtual objects. [61].

These three parts must be accounted for for a precise calculation of the radiance that is outgoing at a particular location on the virtual object. The first part could be sampled directly from the real scene by capturing or estimating the light conditions. The other parts are computed recursively utilizing the global illumination algorithms [83].

Additionally, the third part must mimic and influence the effects of the synthetic objects as if they were in the real scene. A model is required to describe the geometry of the real surfaces and reflectance properties impacted by the virtual objects. Therefore, usually dividing the real scene into two parts is the typical approach: a distant scene 


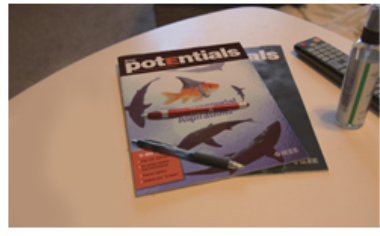

$L_{c}$

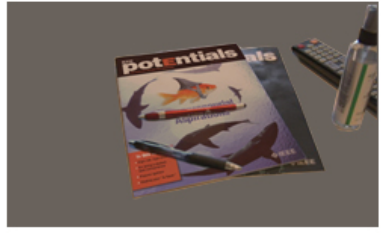

$L_{r}$

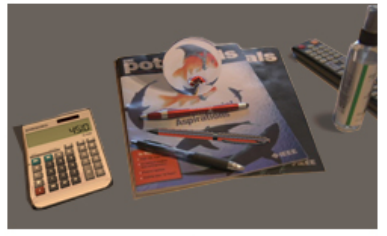

$L_{m}$

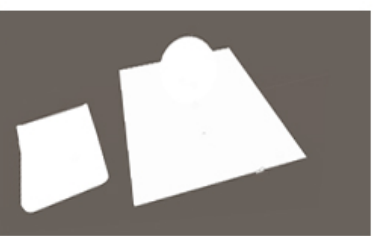

$M$

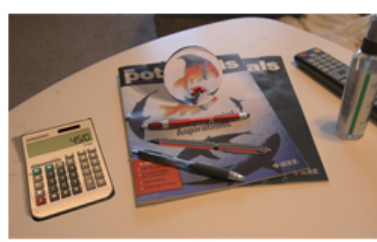

$L_{f}$

Fig. 4. Illustration of differential rendering. [5, 6]

where the virtual objects are not affecting the scene, and a local scene where they are affecting the scene. The lighting simulation is enabled in the local scene model [19].

The interaction among the synthetic (virtual) objects and real scene or objects is computed using a standard technique called differential rendering. The method involves updating the pixels of the background image or video for applying the difference of the reflected radiance as:

$$
L_{f}=M \odot L_{m}+(1-M) \odot\left(L_{c}+L_{m}-L_{r}\right)
$$

Where $L_{f}$ is the final image composited, $M$ represents the rendering alpha mask of the first image created [ 1 if the pixels overlapped with a virtual object, or 0 if they overlapped with a real object]. While $L_{m}$ stands for the resulted image after rendering both real/virtual objects with mixed radiance, $L_{r}$ is the image that represent the real objects rendered with the real radiance. Finally, $L_{c}$ refers to the source image or video taken by the camera. The symbol $\odot$ represents the Hadamard product of the respective color vector [48, 61], see Figure 4.

\section{ACQUISITION OF LIGHTING METHODS}

This section explores multiple techniques that explicitly acquire the lighting conditions and illumination aspects from the real scene where the virtual objects are located using tracking algorithms.

\subsection{Image-Based Lighting (IBL)}

A single omnidirectional image known as an environment map was utilized to represent the incident radiance at a certain point of the real scene. This procedure captured the angular distribution at that single point. During rendering, the captured panoramic image which is also called a light probe is used to recreate the real physical lighting conditions on the virtual scene or objects. We can see in Figure 5 how to capture the scene source light while rendering using the panoramic HDR image.

Any pixel in an HDR environment map can be considered as a lighting incident measurement over an angle solidly subtended by the same pixel. The virtual object's coordinate system must be aligned with the environment map. This Manuscript submitted to ACM 


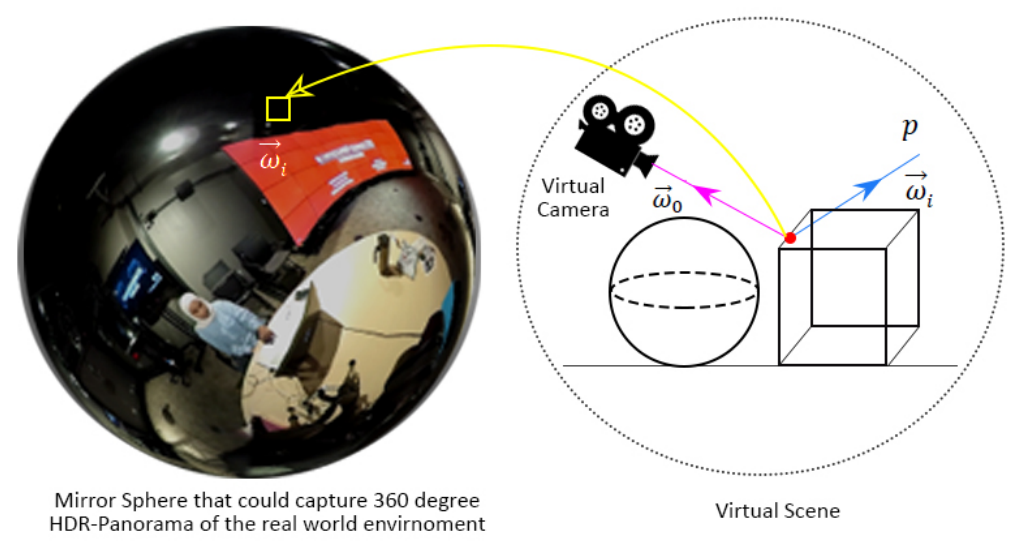

Fig. 5. Allocation in HDR environment map and spherical projection concept. [3, 4, 61]

allows us to interpret a ray from a pixel not intersecting the virtual object that is aligned with coordinate system while sampling the lighting condition.

In earlier work, to simulate the perfect specular reflection and refraction, the panoramic images were used. At a surface point, the reflected or refracted vector could be used for specular scattering events as a direct lookup in the environment map. The method encounters some limitations as it is able to handle only the specular effects, and also requires the environment map to be preprocessed with a low-pass kernel using the surface normal to lookup the reflected radiance.

The first person who proposed integrating arbitrary BRDFs was Debevec [19] who also incorporated global illumination effects, and established studying the relationship/interaction among the local parts of the real physical scene with the synthetic objects. He employed a HDR environment map for capturing the real scene incident light. The full range of light can be captured using the HDR imaging as linear response measurements [55]. The light conditions of the real world can be represented accurately by measuring or estimating the full dynamic range in the scene directly. For further reading of the HDR image capturing the reader is referred to [25, 86].

The idea behind capturing omnidirectional HDR images was practical and straightforward. The method was based on placing a mirrored sphere where incident illumination needs to be obtained in the scene. Then, as many images as required are captured for the scene HDR photographs using a standard lens camera. The reason for taking multiple pictures is to cover all blind spots and to improve the resolution because a single image includes only the area in front of the camera resulting in reduced resolution. However, this system has one problem, which is that the directions were measured from different points in space, i.e., there is no central projection point. For accomplishing this objective, some studies used parabolic or hyperbolic mirrors but were rarely used for real-life applications [2, 54]. Also, capturing omnidirectional HDR environment maps could be achieved using fish-eye lenses, specialized hardware and cameras, and panorama stitching [10, 12, 47, 48, 54, 87, 89, 96].

In an outdoor scene, the illumination conditions usually revealed an actual high dynamic range that makes it necessary to capture images of the sun and the sky using a number of techniques that utilize an accurate combination of aperture, exposure time, and filters [2, 36, 66, 109]. 


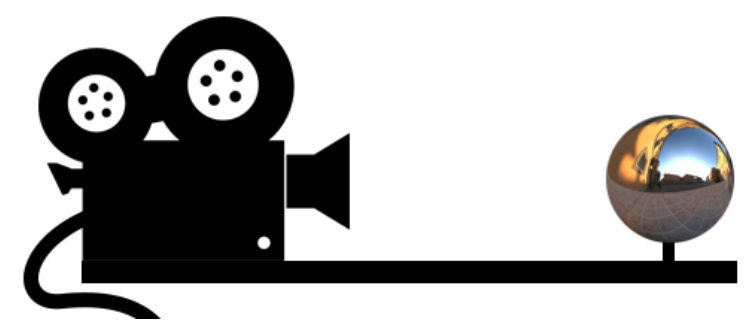

Fig. 6. Temporally variant image based lighting example setup.

The ambient light is usually exceeded by many orders of magnitude in the HDR images which are vital for capturing the light source. However, it could be time-consuming, and it can complicate the real-time capturing because it requires capturing, assembling, and aligning the range of images captured at different exposures. To overcome this obstacle, there was an option of using inverse tone-mapping algorithms for high-quality lighting which is based on linearizing and expanding the dynamic range of panoramic low dynamic range (LDR) images [61, 96, 97]. However, overexposed areas can be washed out resulting in information loss, thus making accurate reconstruction more challenging in large and overexposed areas.

Some studies indicated that the intensity of one bright light source in the scene could be estimated by placing a diffuse physical sphere in the scene and analyzing the captured image. While it is known that the mirrored sphere presents more accurate illumination, at least two images are required to estimate a single saturated light source [10, 20, 44].

Debevec et al.[20] used a novel design of light probe as a mirrored sphere divided into quadrants by diffuse strips. They demonstrated how to recover the full dynamic range of scene from a single exposure. The intensity of several saturated light sources could be estimated based on individually shot light probe image by a simple linear system solution.

A different approach is to set the intensity manually by extracting saturated regions of an LDR light probe. A more intuitive representation to work with is to convert the light probes into a sphere for the directional lights.

\subsection{Temporally-Variant Illumination (Video Sequences)}

An accurate illumination and temporal consistency can also be accomplished in the synthetic object composited into real-world video sequences. The challenges of capturing the light probe at dynamic video frame rates exceed those of static scenes. Therefore, to address these challenges, multiple methods have been suggested to overcome the problem and produce acceptable results. Some successful methods used high dynamic range (HDR-video) cameras in real-time attached to typical light probes and typical video cameras with distinctive optical filters or customized light probes [54, 61, 89, 96, 99, 106].

Knecht et al. [54] captured the surrounding environment and incident illumination by using a fish-eye camera where the system uses a pre-modeled real scene representation. They simulated some direct incident light with a small pocket lamp, then used the Studierstube Tracker to track the camera and lamp position. The default representation is 256 virtual point lights (VPLs) and 1047 points per VPL for the scene. However, the results need some improvement in order to solve the wrong double shadowing and inconsistent coloring in some cases, and also a different shading method is used to increase the image quality. Kaufmann et al. [48] also captured the light in the real environment utilizing a fish-eye camera which allows the estimation of light source position and intensity.

Manuscript submitted to ACM 
Rohmer et al.'s [89] work involved extracting illumination information around the indoor environment by placing several HDR video cameras in the near-field environment. The extracted information was applied to the virtual object to simulate the current lighting condition where that object can change position freely with a consistent simulation of the illumination effects adapting to temporal deviations. The study took advantage of the distributed systems using a tablet camera and stationary PC where the source of light is not visible. Their work required external tracking and was designed for diffuse lambertian objects, but it also provided a plausible display on glossy materials.

Unger et al. [106] formalized the transition of the old use of one or possibly multiple filtered frames of HDR panoramic to the use of HDR-video input. They adopted techniques from pre-computed radiance transfer (PRT) to extend the dynamic processing and rendering of the real scene as input instead of a simulated video, see Figure 6.

Liu et al. [66] suggested tracking the illumination in the outdoor environment online using a complete image-based lighting method which varies from capturing a video with moving cameras. The intensity of the sun/skylight was captured by an optimized process to provide coherence in the temporal illumination. They used a set of real-life videos to demonstrate the visual coherence of the results along the video sequences. This study also covered the spatial variations and the estimation procedure.

Son et al. [98] obtained information about the light from the surrounding environment using the omnidirectional camera that has a typical low dynamic range (LDR) sensor to improve time-consuming in HDR. Furthermore, the light information can be transmitted to mobile devices to generate a realistic composition scene. Over the video sequence to generate a realistic result, the coherence between the spatial and temporal light should be maintained between the background and synthetic objects.

Gruber et al. [36] presented an approach that can handle the dynamic change of light sources and scene geometry with one portable RGB-D sensor in real-time. Temporal consistency is an essential aspect of rendering with temporallyvarying illumination. A significant degree of visual noise is expected while depending on the light probe sequences as an input method. Temporal filtering or special rendering methods can fix the light probe sequence noise [12, 74].

Franke [27] introduced a relighting algorithm which computes the direct shadows and first bouncing indirect illumination called Delta Voxel Cone Tracing. The algorithm is temporally coherent and combined real and virtual surfaces with the extracted illumination at interactive rate.

\subsection{Spatially-Variant Illumination}

There is a well-known limitation for the traditional image-based techniques regarding capturing the light when it varies based on the different locations in the real scene, i.e., illumination with spatially-varying features. Shadowcasting and light shafting were factors in lighting designs which are used in visualization and cinematography production. There is a noticeable difference between the traditional rendering results of image-based lighting using omnidirectional light measurement, and rendering with a spatially varying scene illumination. Even though the result of conventional IBL rendering using a single HDR environment map looks realistic, it does not capture significant details about the scene lighting [105].

Spatially-varying lighting environment $L\left(p^{\prime}, \vec{w}_{i}\right)$ measurement and representation requires capturing the scene lighting with angular distribution at several locations, and/or capturing the scene structure such as depth, parallax as a geometric model. These techniques are based on making assumptions and provide results in a different way. The amount of light measurements can vary greatly depend on using one HDR map or millions of samples. Also, the quality and accuracy of the reconstructed 3D geometry with details varies significantly by which computer vision algorithms 


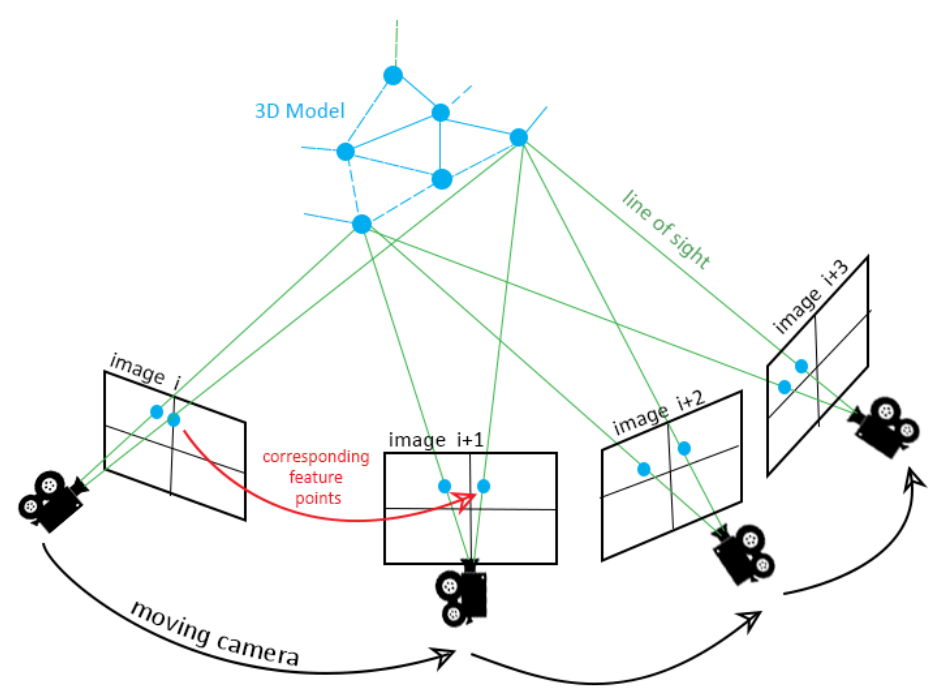

Fig. 7. An demonstrator of structure from motion (SfM) concept.

was used, for instance, laser scanning, structure from motion (SfM), see Figure 7, or various pose estimation algorithms or others $[11,87,105,106]$.

Although using complex techniques could provide better accuracy, there is an obvious trade-off involved with these techniques such as processing time and user interaction. The amount of work and time spent by the user and the type of application are the factors that could determine the choice between the less complicated or more accurate methods. Most of the techniques produce plausible results. However, the methods that are less involved could result in a cruder illumination/lighting environment $L\left(p^{\prime}, \vec{w}_{i}\right)$ approximation. Therefore, the spatially-varying illumination sampling [61] techniques could be divided into two main categories:

- Dense (heavy) point samples with no or a minimum number of geometric techniques that practice a significant amount of angular and spatial radiance samples to represent crude/no geometric scene model.

- Sparse (light) point samples with coarse geometry techniques assuming only Lambertian surfaces is the real-scene using a minimum number of omnidirectional HDR environment maps and representing a coarse geometric model for the scene.

Within each category, we will discuss some techniques and methods of spatially-varying image based lighting.

4.3.1 Dense (heavy) Sampling. The techniques reviewed here are used for capturing and representing a subset, or part of the incident light field (ILF) of some synthetic objects placed at a specific region of the real scene during rendering. The ILF concept is related to the "light fields" notion in photography. The aim of this notion is capturing and processing the reflected/omitted outgoing light-field as small sections of the real scene being captured as photographs. This concept facilitates the applications development of depth estimation, post-capture refocusing, and slight viewpoint transformations [41, 52, 61].

Manuscript submitted to ACM 
However, the ILF goal is totally the opposite to the light fields in capturing the light incident onto a certain region of the scene where interpolating nearby sampling points can estimate the full dynamic range for spatial and angular lighting variations $L\left(p, \vec{w}_{i}\right)[73,89,106]$.

Although most $\mathrm{AR}$ techniques are based on $3 \mathrm{D}$ and six degrees of freedom manipulation of real objects, there are times when $1 \mathrm{D} / 2 \mathrm{D}$ procedures are required.

One-dimension spatial variation. Some of the old experiments used a set of dense HDR environment map images in the 1D path using an HDR-video camera from 1D light probe sequence to accurately capture and reproduce the spatial variation details in rendering. The difference between the rendering method used by Unger et al. [104] and the single HDR light probe environment lookup is the influence of the environment on the point of incidence instead of using the incident direction. The dataset enables the ability to capture light variations that cannot be handled using the traditional IBL, which is evidence that a light field of spatially-variant illumination could produce a useful and powerful extension for the image-based lighting [104]. Most 1D algorithms in AR are used for tracking and interactions techniques. For more details, the reader is referred to $[16,108]$.

Nowrouzezahrai et al. [80] captured the environment lighting using a mirror sphere. They computed $L_{\text {out }}\left(p, \vec{w}_{o}\right)$ which is the shade at a certain point $x$ toward the view direction $\vec{w}_{o}$, which required the distribution of incident lighting at $p, L_{i n}(p, \vec{w})$. The study assumed the spatial variation of lighting could be aggregated into the directional distribution which performed as an environment map of incident light where $L_{i n}\left(p, \vec{w}_{o}\right)=L_{e n v}(\vec{w})$. The process of shading the virtual objects with real scene lighting increased the probability of consistent appearance among the virtual and real objects.

Two-dimensional spatial variation. Bradley et al.'s work [13] focused on aligning the virtual augmentations with a un-rigid plane or on flexible real objects such as cloth. They included an image-based approach for an automatic probing of the real-world light and shadow from HDR video. A 2D textured mesh rendering process aligned with the surface of the cloth and combined with the illumination result to achieve a non-rigid augmentation. The effect of diffuse reflection, ripples, and wrinkles can be seen in most of the cloth results where there is spatial variation in lighting. Therefore, if we replaced the diffuse light and shadow on the augmented objects, they could achieve a realistic output.

Frahm et al. [24] used fish-eye cameras and a TV camera for capturing the source of light in two images because the image facing the camera usually escapes the light source. In these images, the light appeared as saturated regions, so the image sequence can be exploited for estimating the spatial samples of the light source. The image region is segmented automatically after the light sources segmentation. The mean assessment of the point samples belongs to the source of light, and the variance of these points are computed by the automatic segmentation. The light position in the reconstructed coordinate system is triangulated from different locations based on the light source. The reconstructions scale rotation of both cameras is subjugated to estimate the light source position which transformed into the front-camera coordinate system for more realistic augmentation.

Three-dimensional spatial variation. The concept of measuring and rendering the incident light-field expanded to capture spatially varying illumination in 3D. Unger et al. [106] reconstructed a geometric scene model where the radiance information captured from the panoramic HDR video sequences with/without mirror sphere is re-projected, then stored as HDR texture to represent the direct sources of light and the surface of the scene.

Each image position and orientation is estimated using external tracking systems. For tracking purposes, they used a $1.5 \times 1.5 \times 1.5 \mathrm{~m}$ translation stage along with an accuracy order of $0.1 \mathrm{~mm}$ and optical tracking based on tracking markers and external cameras. They computed two different data sets based on the image data. First, the dense point cloud that describes the scene geometry is estimated by structure from motion techniques on the HDR video frames. 
Second, the volumetric dataset of the illumination variations is computed from panoramic HDR video sequences which called focal volume. Each HDR-panorama pixel corresponds to a radiance sample which is picked from a particular position and direction in the scene.

The 3D dataset enables the user to improve the rapid estimation of the point cloud or model parts manually. The focal surface is placed inside the volume. The final geometry recovered from the scene reveals the robust light sources as high-intensity cones in the volume. The distinct voxels segmentation that corresponded to each light is enabled for position extracting and spatial extending using spatial selection and thresholding. Finally, the radiance samples are re-projected onto the recovered geometry that was stored as HDR textures. The recovered model during rendering of a point on an object is used to estimate the incident radiance.

4.3.2 Sparse (light) Sampling. The complete capture of spatial variations of illumination measurements may not be practical in various situations. In rapidly changing environments like a film set, the changes should be captured prior to the next light scattering when the set re-organized. The techniques are used for this purpose to capture smaller samples of the HDR environment map such as one or two, only to enable high-speed and economical light capturing. Therefore, these methods are considered valuable in dynamic environments.

Computer vision and geometric relations are exploited by many of these methods to recover geometric scene information and provide spatial variation in the lighting. Lambertian surfaces are the default assumption of what the subjects are made up of in most studies. Even with the reasonable quality to capture the spatial variations effects, some of these assumptions are not as realistic as the other advanced methods that we will cover next.

One of the first methods was proposed by Sato et al. [92]; they used two omnidirectional cameras to generate the environment spatial radiance distribution utilizing stereo matching. The lighting information is presented as a 3D mesh which is used in the rendering process as an area of a light source which provides spatial variations in illumination.

Corsini et al. [18] suggested stereo light probes that used dual HDR environment maps instead of one. They used the spherical stereo to acquire not only the light sources direction and intensity but also the concrete position of these sources in the space [60].

Happa et al. [38] improved the lighting method in 3D modeled/scanned environment. Their approach relights synthetic interior scenes by IBL extension for generating great quality and fast interactive previews of the environments. It required light probes placed in the real scene, then manually aligned with the 3D mesh of the environment. Ultimately, the light is emitted from the HDR environment map has similarity to the instant radiosity or the photon mapping. Cook et al. [17] also present a method for the real-time photo-realistic rendering of photographs with synthetic objects in interior design by sampling each frame for diffuse shading to illuminate the virtual objects.

EnvyDepth developed by Banterle et al. [8] is a more general system which enables the user to paint or splatter depth onto an HDR environment map to generate a depth map using geometric constraints on primitives like planes, curves, and domes. From that understanding, this tool takes advantage of propagation editing for generating a detailed assemblage of virtual point lights which re-produce effects on both distant and local lighting in the real scene. The spatial information can provide more simulated effects such as shadow, highlights, and caustics compared to using single light in the distance. Therefore, without the struggle required for creating precise scene reconstructions, and without visible artifacts, EnvyDepth takes a few seconds only to produce plausible lighting.

A structured importance sampling is a technique introduced by Havran et al. [39] who were the first to use HDR video camera to render illuminated scenes by distant light based on Monte Carlo sampling. 


\section{REGISTRATION AND ESTIMATION OF LIGHTING METHODS}

Registration is a transformation for the obtained sets of data which are the characteristic geometric and photometric properties, such as data obtained from sensors, day-time, depth maps, or perspectives, into a single coordinate system of the real/virtual scene. The process is required to compare and integrate the data acquired from this measurement.

The real scene physical measurements such as light probes and other devices are considered repetitive requirements and time-consuming. Therefore, images and videos are not acceptable as physical measurements in the real scene for this section. Many previous studies have concentrated on mining approximate lighting data directly from the images or video feeds to avoid these measurements in the real scene [61].

The direct lighting computation on a regular image is generally an ill-posed problem driving to the similar observation on that image with many potential solutions. Therefore, environment assumptions should be considered, for instance, known scene geometry, Lambertian reflectance, or observing illumination distribution [95].

The methods are categorized in this section according to the recovery procedure of the incident illumination from the original scene.

\subsection{Explicit Geometric Registration}

The methods used in this section are based on the reconstruction of scene geometry in a detailed manner which is usually recovered utilizing computer vision techniques, laser scanning, or manual modeling. The lighting data are estimated either using a few HDR images or several HDR environment maps, which could be represented as 2D texture mapping or surface light-fields $4 \mathrm{D}$ function that is projected onto the scene geometric model [61].

It might be challenging to estimate and represent most of the spatial variations in scene illumination, like a sharp shadow, light shafts, and parallax effects accurately without estimating the 3D geometry of the scene. Nevertheless, many of these effects could be available if an accurate model has been recovered from the scene.

Debevec et al. [21] proposed a laser scanning system to capture an outdoor scene environment based on recovering geometry, capturing textures and lighting measurements. The 3D model projects the system textures and measurements of lighting to estimate the material properties at the scene surfaces using inverse global illumination techniques. The synthetic rendering output presented is taken from the photograph and by generated lighting setup.

Unger et al. $[105,106]$ presented a system for capturing, processing, and rendering virtual photo sets. The pipeline for capturing is image-based relying on SfM methods with dense geometry, and interactive tools set to estimate, and semi-automatically adjust the recovered scene geometry. The virtual photo set model composed of 3D geometry where the light information was captured from the projected HDR video sequence. The lighting data stored as a form of 2D textures or 4D surfaces light field. The paper explored tools used to estimate the sources, position, and orientation of the light in the real scene, and the method to estimate the BRDF on surfaces with dense samples in the recovered model.

Meilland et al. [75] described another system that was involved with real-time 3D mapping and tracking using an RGB-D camera such as Kinect for recovering an irregular geometric scene. The observed dynamic range is employed for estimating the camera pose and the density of the scene structure which fuse LDR exposure into light fields of HDR surfaces. The laser scanning is commonly used to recover scene geometry by surveying landmarks or hand modeling for visual effects productions. Recently, the development of captured/painted HDR texture tools has become more critical for realistic results [61, 106].

Manuscript submitted to ACM 

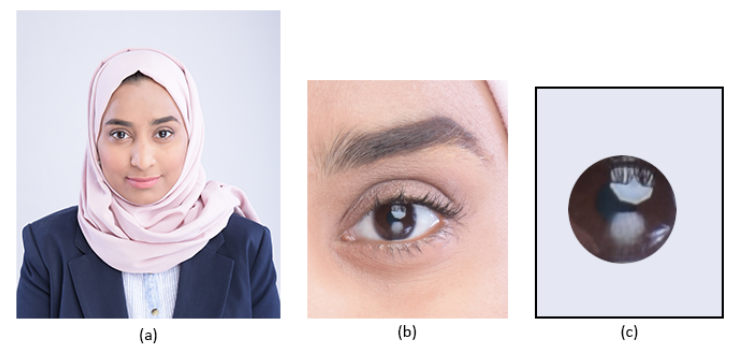

Fig. 8. (a) A frontal headshot. (b) A magnified eye cornea. (c) Using the eye pupil as an environment map.

\subsection{Photometric Registration from Implicit Geometry}

Familiar objects in several scenes with known or trivial geometry can be employed for estimating the incident illumination.

The notion of using the human eye as natural light probes was observed by Tsumura et al. [103] Then, Nishino et al. [79] took advantage of this observation to develop a robust framework to estimate the incident illumination by observing the eye cornea and acquiring the reflected scene radiance, see Figure 8.

Moreover, using the characteristic of the human face to estimate the real-world lighting condition was also exploited by Knorr and Kurz [55] to propose another framework. The model of face-appearance is explored from multiple faces dataset that was loaded offline under pre-known lighting conditions. The most acceptable lighting conditions of real-world in the bases of spherical harmonics are recovered at the run-time.

Some studies employed RGBD cameras, such as the Kinect sensor to approximate and update the scene geometry dynamically. The low frequency of incident illumination can be recovered based on the Lambertian scene assumption which reflects temporal variations [11, 40,74].

\subsection{Photometric Registration from Specular Reflections}

The incident light can be estimated from the reflected direction by observing the specular reflections on pre-registered objects. The concept can be used on any specular object in the scene with a recognizable shape without any constraints to any light probe.

For instance, Jachnik et al. [41] presented an approach to capture the incident light field from a certain surface by a particular camera with tracking browsing ability. They used surface light-field instead of the more popular two

plane light field parameterization. The surface of light-field can be denoted as a $4 \mathrm{D}$ function $\left.L\left(p, \vec{w}_{i}\right)\right)$, where the planar case $p^{\prime}=(p, d)$ is the texture element for 2D position in a simple Cartesian coordinate system. The radiance function depending on viewing direction was utilized instead of using a single RGB radiance value for texture element of particular surface. The function represented by discretizing the hemisphere surface and the value of a discrete point are stored. The hemisphere samples need to be spread evenly as much as possible for an efficient representation of the outgoing light distribution [41].

\subsection{Photometric Registration from Diffuse Reflections}

The attempt of computing the photometric registration from diffuse reflections seems viable when no specular reflections can be identified in the scene. The more common approach for indoor scenes is the diffuse surfaces. The more difficult type Manuscript submitted to ACM 


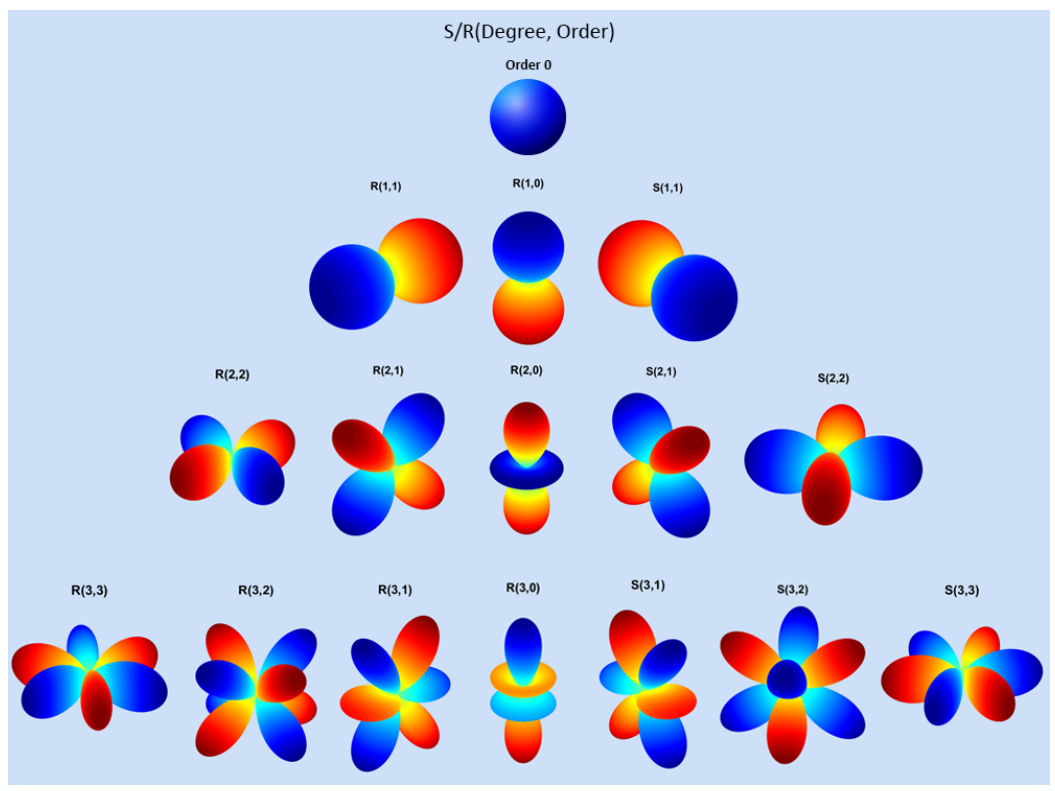

Fig. 9. The first four orders 0, 1, 2, 3 of Spherical Harmonic function based on the origin and color distance on a sphere unit, Blue represents the positive values, and red represents the negative one.

of inverse rendering problem is incident light recovery from diffuse surfaces because separating the light contributions from different directions is crucial. Usually, the estimation only has a single dominant light direction.

Stauder [100] proposed the first video conferencing system capable of autonomously estimating an ambient light and single distant point light by estimating the scene geometry. The system obtained an estimate of directional light by estimating background segmentation of ellipsoid geometric models. Furthermore, Illumination can be stored in a more mathematical and consistent approach by using spherical harmonic $(\mathrm{SH})$, which represents a 2D function on a sphere over all possible directions as the basis-functions set in a form of linear combination, see Figure 9 [85].

The low-frequency representation is usually sufficient for storage because a few numerical coefficients per cache entry are needed to be compressed in SH form. Furthermore, The SH form to compute the diffuse light transport is very inexpensive and can be stored in a surface texture map [53].

Gruber et al. [35] demonstrated the SH framework ability for recovering lighting in the real scene in real-time by employing only an RGB-D camera. The images of depth are used to reconstruct the scene. The directional incident light was solved by assuming only the SH form of diffuse reflection from sample points on the reconstructed surfaces. This distribution of surface normal should be represented efficiently with good sample points. While the diffuse reflection collected the light from every direction, a shadow from other objects should be computed for each sample point in the scene.

Also Gruber et al. [36] represented a desktop GPU system with optimizations of image-space that can estimate the light source and the shadow cast from a dynamic object with $20 \mathrm{fps}$. Also, Boom et al. [12] proposed a complete system for estimating one light source from the arbitrarily geometry of a scene assuming diffuse reflection for the whole scene. The method concept is an image segmentation based on color into super-pixels for assumed constant albedo also known as diffuse reflectance. The light source can be recovered with reasonable accuracy as long as the albedo is known. 


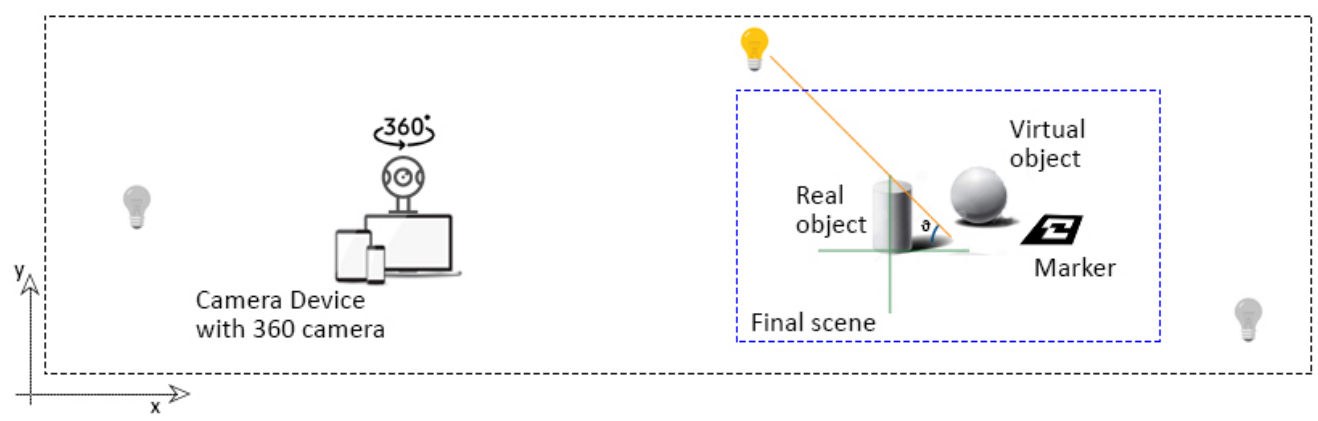

Fig. 10. Estimating the incident light direction by forming a ray from a unique point of the contour of the shadow that is a corresponding surface point on the shadow caster. [5]

\subsection{Photometric Registration from Shadow}

The light source can also be estimated using another method, which is by observing the shadow in an image, see Figure 10. The full or partial knowledge of the shadow caster's geometry, the correct classification, and the shadow appearance measurement in an image are the principles of this method.

The visible object geometry boundary of the shadow caster can be traced back by surface points on the contour. Therefore, estimating the direction of single or multiple light sources is applicable [95].

A complete overview of most known algorithms and methods based on shadow volume in computer graphics are discussed in detail by Kolivand and Sunar [56]. The algorithms were divided into two subsections: image-based and geometry based.

Haller et al. [37] represented a special geometry characteristic employing a light probe called shadow caster that captured light from any direction. The more challenging approach is detecting shadows in natural images [11, 75, 97, 109].

As is well known, shadows are caused by the occlusion of incoming light in the real scene by objects. Therefore, in order to estimate the illumination distribution, the relation between the image brightness and the occlusion of incoming light can be analyzed. Sato et al. [93] introduced a technique to estimate the illumination distribution by investigating the image brightness inside shadow cast from a known shape object.

\subsection{Photometric Registration from Images}

The problem of inverse rendering was mentioned in details by Patow et al. [82] where a curved object is photographed from various angles to capture the reflected light from a different orientation of the surface.

Marschner [71, 72] presents a system called image-based BRDF to measure reflectance quickly without any additional equipment that only required a series of photographs using a digital camera and stable light source.

Although Reconstructing real scene geometry from photographs using several viewpoints was implemented by Loscos et al. [68], a different method to recover reflectance was presented using controlled and fixed viewpoint to capture a set of images. A varying illumination without shadow and confidence weight factor are combined to represent the visibility under the light source consideration for every pixel in the images. The effect of shadow was considered later in [67] which presents an algorithm for interactive relighting based on geometry reconstruction and not occluded illumination textures creation in a reprocessing step.

Manuscript submitted to ACM 
Poulin et al. [84] introduced a three step process for 3D geometry reconstruction and texture extraction through an interactive system using a set of photographs. The authors solved a least-squares problem for the camera parameters first, then for the 3D geometry. The color textures of 3D model are extracted through re-projected texels sampling, when a satisfying model is retrieved. In order to form a unique texture, a fitting process is applied for all the textures then a combining process to the corresponding colors is achieved under certain criteria. Sato et al. [94] also used a sequence of images and a reconstructed 3D model to recover a simplified BRDF model know as Torrance-Sparrow reflection model for an isolated object.

On the other hand, Mandl et al. [70] proposed learning light probes that use pure synthetic images which are then applied on real image data-set to train convolution neural networks (CNN) for high-quality illumination estimation. This method was developed to reduce the run-time in the inverse rendering techniques. Weber et al. [107] developed a deep learning method which was trained on a database of environment maps to estimate indoor lighting given a single image of a known 3D object.

Gardner et al. [28] trained a lighting classifier that is robust and annotates the light location by automatically using LDR environment map dataset. Then these annotations were used to train a deep neural network for light location and intensity prediction in a scene from a single and limited field of view image.

Morgand et al. [77] presented a geometric model which is reconstructed from images of specular material which depict the light sources on the planar surface to predict the shape of the specularities and the light source.

Grosch [33] combined inverse rendering for light reconstruction and differential rendering for displaying the changes to an interactive tool to maintain consistent lighting with real-time modification using panoramic images.

\subsection{Photometric Registration for Outdoor Scenes}

Typically, the scene's complete geometric model in outdoor AR systems is not accessible, which creates a more challenging photometric registration. On the other hand, during the daytime, the simple strongest light source is the direct illumination from the sun which drives the simple illumination model. The first estimate uses the analytical model of the sun. The light source of a large area such as the sky can be a secondary approximation. Shadow cues can be used in the image for enhanced outcomes [10, 65, 66, 99, 109].

Lalonde et al. [62] presented a method that calculates the probability distribution over the position of the sunlight and its visibility to estimate the scene illumination conditions using a single outdoor image from a large data set of internet photographs.

Madsen et al. [69] assumed an outdoor scene to automatically detect the shadow in the image then determine the relation between the sky irradiance to the sun irradiance. The sun position was computed based on the date, time, and location on the earth.

Kolivand et al. [57,59] proposed a new and unique technique for realistically rendering the outdoor scenes in real-time by taking into account the sky color with respect to the sun position which involve with shadow generating algorithm, Z-Partitioning: Gaussian and Fog Shadow Maps.

\section{COMPOSITION AND GLOBAL ILLUMINATION METHODS}

The final and single image resulting from merging the virtual objects into the real scene called composition. In this part, we will cover the real-time global illumination methods that face two main dimensions of complexity.

The first dimension is the type of light transport which is being simulated. The simplest class of algorithms is a shadow which only allows the removal of light. Soft shadow and color bleeding are permitted by the diffuse global 
illumination where the light is strongly reflected off colored surfaces to nearby objects. The ideal addition is to apply well-known specular effects, for instance, reflection, refraction, and caustics, for selected objects. Thus, the highest complexity for the entire scene is allowed arbitrary diffuse and specular light transport.

The second dimension is concerned with the scene; all light transport can be pre-computed for static lighting in a static scene where the camera is the only moving part. Pre-computation could solve the online performance problems. However, it may require excessive computational storage and resources, particularly if the system supports specular effects. On the other hand, it is necessary, at least, to compute the effect of dynamic objects on the light transport for every frame in the scene. Thus, the highest computational cost in a scene is when it has dynamic objects and dynamic light transport [95].

The computational cost for the global illumination is determined by these two dimensions, in addition to the scene size. In large dynamic scenes accompanied with complicated light transport features, the real-time updates still depend on high-performance workstations and mobile devices.

Two rendering passes factorization are introduced by many modern global illumination methods for the more tractable approach. Several advantages are gained by this separation where the scene light transport is computed in the first pass, while the distributed light information which forms the final image is collected in the second pass.

More information about rendering is discussed in section 7. Currently, this section is organized by the light transport complexity beginning with cast shadow on the scene, followed by diffuse global illumination to specular global illumination.

\subsection{Shadows in Common Illumination}

The 3D scene structure could be determined mentally by a human observer using the shadow cues. Sugano et al. [101] addressed the visual consistency where the lighting and shadow of virtual objects should match the real objects in AR scenes to increase the perceived realism. It is an expensive cost to compute reflection and shadow simultaneously, therefore computing the shadow can be an acceptable alternative. The representation of a shadow can be computed in the first pass, while this representation could be used for the shading of the surface points of the final image in the second pass. The existent shadowing techniques are influenced by shadow volumes and shadow mapping [24, 42, 88, 99]. For objects enclosed in a frustum which lie in shadow with respect to a given light source and shadow-casting polygon, that frustum is known as shadow volume, and its sides are called shadow volume polygons [95].

Everitt and Kilgard [22] presented a shadow volume technique based on the standard feature of the GPU called the stencil buffer. The technique of shadow volumes has four passes. (1) Draw the scene without illumination (i.e., the scene in the shadow). (2) The front facing shadow volume polygons rasterization discretely increases the stencil buffer. (3) The back facing shadow volume polygons rasterization discretely reduces the stencil buffer. (4) redraw the scene with every fragment that has zero value in the stencil buffer where the fragment is rendered illuminated and not in the shadow.

Other considerations that must be taken under common illumination include not only real object shadows or virtual object shadows separately, but also a consistent shadow cast from real to virtual and the other way around. Haller et al. [37] developed the technique presented by Everitt and Kilgard to apply for the common illumination. In the first pass, render the shadows that are casting from virtual objects to the real ones. In the second pass, render every virtual object including received shadows from virtual or real objects.

When the video feed initializes the frame-buffer, simultaneously the phantoms are rendered at first pass to the z-buffer, where the stencil buffer contains the shadow volumes of the virtual objects. The shadow cast from these 


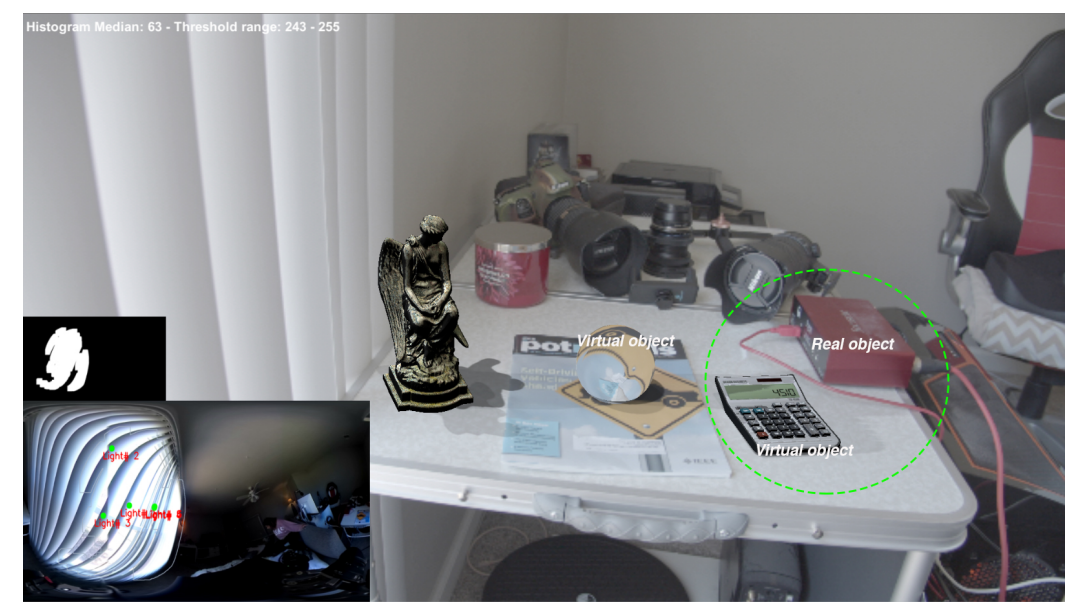

Fig. 11. Double Shadowing problem occurred when adding a virtual shadow of a virtual object (calculator) on a region in a shadow already from a real object (sound mixer) $[3,5,6]$

objects to the real ones are created using the stencil buffer. All pixels of the video are blended to create an impression of the shadowed region by marking the stencil mask with a dark transparent color.

The second pass compares the traditional rendering of the stencil shadow volume. While the whole scene is rendered into the color buffer, the shadow volumes are drawn into the stencil buffer. A mask from the resulting stencil buffer then is used to redraw the whole scene with ambient and emissive components only, which makes the objects that fall into the shadow ray unlit by any light source available [2, 24, 95].

Shadow mapping technique is used in building the most contemporary rendering systems due to the fully accelerated texture mapping on the GPU. There are two passes for shadow mapping technique: in the first pass, the whole scene is rendered from the perspective of the light source to the depth buffer; in the second pass, the shadow map is used to determine if a fragment is occluded also from the light source perspective to be rendered from the viewpoint of an observer. The fragment is considered part of the shadow if its depth in the coordinate of the light source is higher than the shadow map entry [24, 54, 74, 99].

Gibson et al. [29] and Supan et al. [102] and other old studies used shadow map to cast soft shadows from virtual to real objects by imposing shadow maps from a great number of light sources which were estimated using a light probe. In the current studies, the blending approach is applied to create soft shadows that can be pre-calculated even in the dynamic illumination scenes [74, 99].

\subsection{Diffuse Global Illumination}

The full global illumination required not only perfect shadows but also a reflection. Some problematic issues can be eliminated using this approach. One of these problems is double shadowing where the shadow cast duplicates the darkening pixels from virtual to real objects if the blending is faulty due to virtual to real shadow overlapping with real to virtual shadow. Diffuse light transport of global illumination algorithms is the main focus of this section, see Figure 11.

The scene surfaces in the classic Radiosity method are turned into discrete small polygonal patches, then the light transport among them is solved. The first pass is an inherently expensive procedure because it requires global visibility 
computing among several patches. On the other hand, the second pass is a simple rendering process for the illuminated patches. Thus, in the dynamic scenes and the current real-time systems, patch-based Radiosity is hardly used. Fournier et al. [23] implemented the first application using the Radiosity method for simulating the common global illumination but was not in real-time and performed with simplified assumptions.

Currently, most approaches aim for real-time performance at least in the second pass. A broad approach employs the shadow mapping on GPU for computing the direct illumination while simulating the indirect illumination with limited accuracy. For instance, Rohmer et al. [89] presented in their previous work how to store indirect illumination in an irradiance volume. For the first pass, radiance transfer from every possible direction onto the static scene is precomputed and combined into an SH form set of irradiance volumes. For the second pass, the shadow map is utilized to compute direct illumination, while the sum of basis irradiance volumes is weighted by the light intensity obtained in the indirect illumination.

Nowrouzezahrai et al. [80] also presented a light factorization algorithm for simple real geometry scenes. Separating the real-world lighting into two main lights, direct and indirect, is the main contribution of their work. For the direct light, they extracted the sources of point light from image-based lighting and employed the shadow mapping to apply them. The radiance transfer of each object is precomputed and represented in SH form which allowed an efficient combination of indirect light that is also represented in $\mathrm{SH}$ form [53].

Lensing and Broll [63] used a Virtual Point Lights (VPL) approach that is used in Knecht et al. [54] that we mentioned earlier, but they used splatting to apply the light form VPL instead of shadow mapping. Obtaining the dynamically moving geometry with deformable real objects using an RGB-D camera to support moving real objects was the critical contribution of the study. For computing illumination, the depth image with a guided edge-preserving filter used to smooth the massive noise in the depth image resulted in a better surface normal estimation. Beyond the current field of view, no real-world geometry existed, and every light source is virtual. They also presented a fast and novel global illumination method that depict indirect light for both diffuse and glossy surfaces for dynamic scenes in real-time with sparse sampling using 3D geometry from Kinect camera [64].

Franke [26] presented a global illumination technique that used a novel volumetric relighting method instead of surface light transfer. He utilized the light propagation volumes from other studies to represent radiance. The method computed a VPL set, and each VPL contribution is injected into a small volume with SH modeled directional radiance. The light propagation difference is computed before and after adding virtual objects to enable differential rendering.

Gruber et al. [36] estimated the light source by maximizing the prospect of global illumination interaction combining real-time data from three sources: the outside field of view (FOV) static geometric model, the inside FOV dynamic geometric model, the lighting of environment dynamic estimation from reflections observed inside the field of view. Finally, the dynamic changes and occlusions which can be hard to observe outside the field of view were generally omitted. For more reading about the interactive occlusion in augmented reality see the research by Tuceyran et al. [14].

\subsection{Specular Global Illumination}

Specular effects from shiny surfaces, such as metal, and translucent materials, such as glass, are restricted in the view-independent methods described above.

Knecht et al. [54] demonstrated how to compute the specular effects in real-time with their specular extension to Differential Instant Radiosity. Unfortunately, a rasterization approach cannot support arbitrary diffuse and specular combinations of light transport which required a more expensive procedure based on ray-tracing.

Manuscript submitted to ACM 
Grosch et al. [32] introduced the first but not real-time method for specular global illumination. Ray-tracing for a photon mapping with a differential version is used in the first pass. Diffuse or specular is the classification of surfaces. The specular surfaces reflected or refracted the photons, but diffuse surfaces stored them. When a virtual object hits by a photon, an anti-radiance which is a negative amount of light is stored in the place where the photon would hit the real object. Then, ray-tracing from the eye is used in the second pass to produce a final image with reflections, refractions, and caustics affecting real imagery from virtual objects.

Kán and Kaufmann [48] used a related method based on the real-time ray-tracer OptiX proposed in [81] for both passes then combined them with photon mapping. Moreover, instead of using anti-radiance, they enhanced the differential rendering in the second pass with separated shadow rays for virtual and real images.

Shi et al. [97] used the global illumination method to generate more realistic material appearances in both AR and synthetic material design. The differential irradiance caching algorithm presented in the previous paper [48] was used combined with the ray-tracing which support enabling several bounces of the global illumination.

For evaluating the differential irradiance at the records of irradiance cache in one pass, they used Monte Carlo integration in GPU ray-tracing. The GPU accompanied with the NVIDIA OptiX ray-tracing engine provide a parallel power to calculate both the direct and specular illumination [81].

\section{DISPLAY AND RENDERING TECHNIQUES}

Displaying your result is the final step to show the composited image to the user where the rendering methods are required. Most common rendering techniques could be divided into two categories:

- Common rendering algorithms for static or dynamic environment maps which include Monte Carlo rendering, conversion to directional light sources, and pre-computed radiance transfer. More details of the material in this section are available in [61].

- Interactive differential rendering methods which are more involved with augmented reality and the illumination concept. Therefore, we will cover this concept in the following section.

Rendering a synthetic object into existing images has been developed over the years. Karsch et al. [51] proposed a method that creates a physical model of the scene which realistically inserts virtual objects into several photographs without any additional information about the scene measurements. The technique was suitable to render the objects with diffuse, specular, and glowing materials under the lighting conditions in the scene.

Gibson and Murta [30] also introduced first GPU-based AR system at real-time interactive rates using image-based method and sphere-mapping to eliminate and render synthetic objects seamlessly into background photographs.

For further evaluation of the existing rendering methods and techniques in computer graphics, Kolivand et al. [58] provided an elaborated survey on photorealistic rendering. The study aimed to ease the selection of the appropriate method in each system developed by researchers using classification and systematization among numerous methods.

\subsection{Interactive Differential Rendering}

The number of rendering passes that are required in the traditional differential rendering is two: one involved the local model of the real environment, and the other is merging both real and virtual objects. Many regions are rendered twice without any change which rise a question about the visual effect under this approach. The use of a single pass is a more efficient approach where the changes in lighting created by the virtual objects are simulated directly.

Manuscript submitted to ACM 
According to real-world lighting conditions, we can compute the common illumination between any kind of objects using a real scene model, virtual scene, and incident light. The light traveling directly from the source to an object and reflected toward an observer is known as direct illumination. The light traveling from the source to an object and reflected toward another object is known as indirect illumination. A simulation of full global illumination can involve many light bounces between the objects before it eventually reaches the observer. The combinations of the object interactions could be any of these four possibilities: from real object to other real object or to a virtual object, and from virtual object to other similar virtual object or different real object. The composition of real and virtual is based on differential rendering which contributes to visual realism.

Common illumination even with a precise photometric registration would not be perfect because it is not possible to fully interpret all light interactions in the scene. However, it would be efficient to preserve the subtle illumination effects which are present naturally in the real scene final image. The process of allowing the real-world illumination to be preserved is referred to as differential rendering. Fournier et al. [23] introduced the concept, then Debevec [19] developed the formula of differential rendering as follows:

Given the scene geometry, scene material, camera parameters, and light source, we can compute a light simulation $L_{R}$ that corresponds to the original scene without virtual objects. A second light simulation $L_{(m)}$ can be computed after inserting the virtual objects. Any pixels depicting virtual objects can be replaced by $L_{(m)}$. For all pixels depicting real objects the difference $L_{(m)}-L_{R}$ shows the changes that happened to the real objects after adding the virtual objects. Then, the difference can be added as a correction term to the camera image $L_{c}$, see Figure 4 .

Therefore, for pixels with virtual objects $L_{f i n a l}=L_{(m)}$, and for pixels with real objects $L_{f i n a l}=L_{C}+L_{(m)}-L_{R}$ can be interpreted as an error term to simulate the result $L_{(m)}$ for correction of any inaccuracies in the modeling $L_{R}$ of the original scene $L_{C}$. The pixels are brightened if the virtual objects indirectly illuminate them $L_{(m)}-L_{(R)}($ positive) However, the pixels are darkened if the virtual objects cast a shadow $L_{(m)}-L_{(R)}$ (negative).

This rendering could be more challenging if the scene modifications provide relighting which changes the light sources and how that will affect the whole scene and not only the objects. Mainly, the idea is to remove the light source and cause the shadow to disappear from the scene. On the other hand, adding a new virtual light source would be applicable where the light can be linearly combined. Therefore, many methods were enhanced and developed to accommodate real-time global illumination.

Grosch et al. [32] modified photon mapping described in Jensen et al. [43] by using a differential photon mapping render in one pass for both real and virtual objects interaction. Every pixel of the environment map is representing a parallel light source. Thus, the photons are shot towards the virtual object: First, they are uniformly distributed on a disk that has a radius perpendicular to the light direction. Then, If the virtual object was hit by a photon, the next intersection point is calculated using the real geometry and a negative flux is assign to that photon. Otherwise, if the virtual object does not intersect by a photon, it can be ignored, due to unchanged status to the light path.

Also, Grosch et al. [34] suggested a global illumination technique for indoor scenes in real-time using diffuse materials by light probes. The representation of near-field reflected light in the room is updated by using the direct light from outside and a dynamic irradiance volume. The direct lighting also used sampling and shadow mapping.

Knecht et al. [54] presented methods which combined instant radiosity utilizing differential rendering that only needs one rendering pass for achieving the real-time performance in both diffuse and specular objects. Their method was extended for handling the reflective and refractive objects, and caustic effects by assuming the real objects geometry is static and given.

Manuscript submitted to ACM 
Kan et al. $[48,49]$ developed a method for interactive global illumination using photon mapping that allows caustic and reflective or refractive materials. Also, they developed a one-pass differential rendering method in real-time by utilized irradiance caching. This irradiance separated real and virtual objects by analyzing both diverse ray types and intersection situations, which could be helpful in the computing process of differential irradiance. It is known that the real and differential irradiance are stored in the irradiance cache record which then can be utilized on the GPU for irradiance cache splatting. This method has some limitation regarding diffuse materials which required pre-computation stages. However, the results were reasonable for multiple bounce global illumination [50].

Lensing et al. [63] solved the pre-computation stage of a one-bounce diffuse indirect lightning using reflective shadow mapping. Also, to overcome the errors of the depth image, the method used was purely image-based with some guided filtering.

The development of differential rendering extended to mobile devices. Rohmer et al. [90, 91] reduced the computational cost for each light using tile-based rendering, in addition to frustum culling techniques tailored for AR systems and applications. Monroy et al. [76] presented a similar system which work in dynamic environment with the ability to scan the real scene and then projected onto a two-dimensional environment map that contain RGB+Depth data.

\section{DISCUSSION AND COMPARISON}

This section provides a summary and discussion about the whole review and the different methods for capturing and rendering in mixed reality scenes. We select some of these methods and compare them with others and observed each method's requirements, advantages and drawback. The study of limitations is the key to improvement in the future work, and we need to highlight them for the next part.

It is very important to acknowledge that each method is not excluded from the others. These techniques could be included within each other or in a different part of the whole process to achieve the desired realism.

In order to explore each technique and make some comparison among these methods, some criteria were established to recognize the major difference and how that weighed in the final outcome. This major difference in aim, accuracy, and robustness between each method makes it challenging to conclude the compression.

Therefore, based on the information provided in each paper we attempt to restrict some of the useful information to write this section. We believe this indication will be helpful as a future reference for our work and others regarding usability, performance and more. Thus, the criteria are:

- Required data. the prerequisite data that are essential to be obtained at the beginning of the procedure or as part of sub-procedure in the whole system.

- Assumptions. some systems work assuming certain conditions such as the number of light sources, objects position, or the indoor/outdoor environment. These systems address their assumption at the beginning of the procedure because it might not work perfectly if any of them were altered.

- Outcome. the solution provided by the system including the accuracy of the result under the previous criteria.

- Drawback. the limitations of the system based on the required data, assumptions, and outcomes. The future work usually starts by discussing and discovering these drawbacks and the attempt to solve them.

There could be more criteria to cover, but it requires insight and evaluation of each system which seems outside the scope of the current review. We might make an approximation for the time and effort for the rendering and processing, but the result would not be trustworthy without any quantitative data.

Manuscript submitted to ACM 
An overview of these criteria to compare the previous methods from selected papers are presented in Table 1. These papers were chosen based on two factors: (1) significance in their field, (2) provide an insight for our future work.

\section{OPEN PROBLEMS AND FUTURE WORK}

While interactive illumination for photorealistic scenes in augmented reality/mixed reality has been the major focus on many research and industrial issues for many years, still there are many open problems and future work that could be accomplished.

Most current methods are based on a static scene assumption that enables capturing spatial variation in scene illumination which requires significant effort during capturing and processing. Also, developing techniques for estimating material properties on the surfaces in scenes could help find methods to measure and estimate varying spatial illumination. Computer vision improvement accompanied by enhanced capturing devices would highly develop this field. For instance, ARToolKit by DAQRI, Tango by Google [91], Vuforia by PTC Inc, and ARKit by Apple are examples of software development kits that use computer vision to provide more techniques for a robust inverse global illumination which could increase the accuracy of virtual objects simulation that affect the real scenes.

Furthermore, most of these kits are compatible with many development tools that could ease the work, such as Xcode for ISO developer adds these packages to existing IOS projects leveraging IOS rendering technologies, in Android Studio the Android developer can add the kit experiences to existing apps using Java APIs and C++ APIs. Also, it works with 3D engines like Unity and Unreal to build a game or high-fidelity 3D experience, then run this application on IOS or Android devices. Some also support developments of Universal Windows Platform (UWP) apps for selected Intel-based window devices, including Microsoft surfaces and HoloLens.

The generalization of existing methods for photorealistic objects added in images makes them limited. The most problematic area involves complex illumination environments and specular objects where future studies are an attempt to present specific assumptions to solve each problem and create a more robust and accurate physical estimation for illumination environments.

A similar problem that should also be subject to further research is about the photorealistic object augmented in the video to provide a dynamic real-time illumination and features in mixed reality. The primary challenge is ensuring the consistency of estimating the material properties such as color and reflectance, with correct illumination, throughout the video feeds. Both the new and optimized schemes can be developed if the temporal coherency took into account by deriving it from the scene which could increase the challenge to allow different lighting conditions for spatial variation within the scene simultaneously. For example, we could save time and money for making a real-time online application for entertainment productions. Other limitations arise from the LDR content to composite photorealistic virtual objects into images or video. The availability of HDR would open many possibilities for improving more robust and general methods. The inverse tone mapping and recovering HDR lighting conditions from LDR media are some of the rousing methods for future researches.

As we mentioned before, the realistic real-time rendering that could capture and estimate the correct illumination is still an open area for research and development. It includes general interactions among real and virtual objects, glossy materials, dynamic scenes, and global illumination environments. Therefore, developing rendering algorithms for mixed reality especially for mobile devices is a significant part of the future work that will allow extensive embracing of photorealistic augmented reality. For instance, using photorealistic augmented reality systems for easing some challenges among mental health patients who have Parkinson's disease [9], Attention Deficit Hyperactivity Disorder (ADHD) [1], Autism Spectrum Disorder (ASD) [15], and phobia [45]. Improving the psychological presumptions of Manuscript submitted to ACM 
the human brain by making this application more realistic and well-blended with the real world could provide better results in the future.

Industrial organizations have been funding more academic augmented and mixed reality research. Many publications focused on topics that enhanced and served each structure's work requirements. The realistic results in global illumination would support the publicizing and advertising for sale of industrial products in general.

In the reverse process, these methods could be used by computer vision techniques for discovering forged or altered images, and the video and authenticity of these media could be used for forensic evidence, for instance. There are many applications and methods involved in this area, and by focusing on one problem at a time, many goals could be achieved.

\section{ACKNOWLEDGMENTS}

We would like to thank every researcher and author of the original papers and publications for providing information and data that help understand the whole concept of illumination and realism.

Table 1. comparison of the overviewed methods from the selected papers that we covered forcing on four criteria: required input, assumption, outcome, and drawback.

\begin{tabular}{|c|c|c|c|c|}
\hline Method & Required Input & Assumptions & Outcome & Drawback \\
\hline \multicolumn{5}{|c|}{ ACQUISITION OF LIGHTING } \\
\hline \multicolumn{5}{|c|}{ Image-Based Lighting } \\
\hline $\begin{array}{l}\text { P. Debevec, } \\
\text { 1998, [19] }\end{array}$ & $\begin{array}{l}\text { HDR using a light } \\
\text { probe. }\end{array}$ & $\begin{array}{l}\text { Distance scene } \\
\text { without reflectance } \\
\text { model. }\end{array}$ & $\begin{array}{l}\text { Acquire the real scene light- } \\
\text { ing effects with a good time } \\
\text { and effort using differential } \\
\text { rendering. }\end{array}$ & $\begin{array}{l}\text { Limited physical accuracy } \\
\text { there is no central projec- } \\
\text { tion point. }\end{array}$ \\
\hline $\begin{array}{l}\text { Schwandt } \\
\text { et al. } \\
2016[96]\end{array}$ & $\begin{array}{l}\text { Single RGB-D cam- } \\
\text { era LDR. }\end{array}$ & $\begin{array}{l}\text { The light source is } \\
\text { only a white light } \\
\text { on the top of the } \\
\text { scene. }\end{array}$ & $\begin{array}{l}\text { Convincing reflections } \\
\text { from the real scene and } \\
\text { other virtual objects. }\end{array}$ & $\begin{array}{l}\text { Incoherent reflectance on } \\
\text { some surfaces that facing } \\
\text { the camera. }\end{array}$ \\
\hline \multicolumn{5}{|c|}{ Temporally-Variant Illumination } \\
\hline $\begin{array}{l}\text { Knecht } \\
\text { et al., } \\
2010[54]\end{array}$ & $\begin{array}{l}\text { LDR video using } \\
\text { the fish-eye camera. }\end{array}$ & $\begin{array}{l}\text { Available real scene } \\
\text { geometric model } \\
\text { with known martial } \\
\text { properties. }\end{array}$ & $\begin{array}{l}\text { Simulate the direct incident } \\
\text { light and track the camera } \\
\text { and lamp position. }\end{array}$ & $\begin{array}{l}\text { Double shadowing and in- } \\
\text { consistent coloring in some } \\
\text { cases. }\end{array}$ \\
\hline $\begin{array}{l}\text { Liu et al. } \\
2012[66]\end{array}$ & $\begin{array}{l}\text { Set of real-life } \\
\text { videos on moving } \\
\text { the camera. }\end{array}$ & $\begin{array}{l}\text { Access to informa- } \\
\text { tion like daytime } \\
\text { and GPS coordi- } \\
\text { nates. Detect some } \\
\text { planar surfaces. }\end{array}$ & $\begin{array}{l}\text { Visual coherent of the re- } \\
\text { sults along with the video } \\
\text { sequences. }\end{array}$ & $\begin{array}{l}\text { Partial 3D data about the } \\
\text { visible final scene is miss- } \\
\text { ing. }\end{array}$ \\
\hline $\begin{array}{l}\text { Son et al. } \\
2012[98]\end{array}$ & $\begin{array}{l}\text { Omnidirectional } \\
\text { camera with LDR } \\
\text { sensor. }\end{array}$ & $\begin{array}{l}\text { Obtain only one } \\
\text { light source. }\end{array}$ & $\begin{array}{l}\text { Improve time-consuming in } \\
\text { HDR. }\end{array}$ & $\begin{array}{l}\text { Limited computation per- } \\
\text { formance and bandwidth on } \\
\text { mobile devices. No shadow- } \\
\text { ing. }\end{array}$ \\
\hline
\end{tabular}

Manuscript submitted to ACM 
Table 1. comparison of the overviewed methods from the selected papers that we covered forcing on four criteria: required input, assumption, outcome, and drawback.

\begin{tabular}{|c|c|c|c|c|}
\hline Method & Required Input & Assumptions & Outcome & Drawback \\
\hline $\begin{array}{l}\text { Gruber et al. } \\
2015[36]\end{array}$ & RGB-D sensor. & $\begin{array}{l}\text { Static geometry and } \\
\text { illumination for the } \\
\text { real scene, the light } \\
\text { is white. }\end{array}$ & $\begin{array}{l}\text { Handle the dynamic change } \\
\text { of light sources and scene } \\
\text { geometry with more 3D in- } \\
\text { formation. }\end{array}$ & $\begin{array}{l}\text { Slow updating reconstruc- } \\
\text { tion, needs filtering to fix } \\
\text { the noise. Only support the } \\
\text { diffuse light. }\end{array}$ \\
\hline \multicolumn{5}{|c|}{ Spatially-Variant Illumination } \\
\hline $\begin{array}{l}\text { Unger et al. } \\
2009 \text { [73] }\end{array}$ & $\begin{array}{l}\text { HDR video }+ \text { light } \\
\text { probe. }\end{array}$ & $\begin{array}{l}\text { No major light } \\
\text { field variation } \\
\text { in the other two } \\
\text { dimensions. }\end{array}$ & $\begin{array}{l}\text { Capture and reproduce the } \\
\text { spatial variation details in } \\
\text { one dimension. }\end{array}$ & $\begin{array}{l}\text { Limited spatial and angu- } \\
\text { lar resolution. Limited accu- } \\
\text { racy measurements. }\end{array}$ \\
\hline $\begin{array}{l}\text { Nowrouze- } \\
\text { zahrai et al. } \\
2011[80]\end{array}$ & HDR light probe. & $\begin{array}{l}\text { The light spatial } \\
\text { variation could } \\
\text { be combined into } \\
\text { a directional dis- } \\
\text { tribution. Static } \\
\text { geometry. }\end{array}$ & $\begin{array}{l}\text { Consistent appearance, } \\
\text { solve light integration and } \\
\text { dynamic visibility. }\end{array}$ & $\begin{array}{l}\text { Shadow only works on } \\
\text { static virtual objects. Diffi- } \\
\text { culty in computing the soft } \\
\text { shadow from the environ- } \\
\text { mental light. }\end{array}$ \\
\hline $\begin{array}{l}\text { Unger et al. } \\
2013 \text { [105] }\end{array}$ & $\begin{array}{l}\text { Multiple filtered } \\
\text { frames of HDR } \\
\text { panoramic. }\end{array}$ & Stationary scene. & $\begin{array}{l}\text { Adopted techniques from } \\
\text { pre-computed radiance } \\
\text { transfer (PRT) to extend the } \\
\text { dynamic processing and } \\
\text { rendering. }\end{array}$ & $\begin{array}{l}\text { Simultaneously capturing } \\
\text { both the temporal and spa- } \\
\text { tial domains. }\end{array}$ \\
\hline $\begin{array}{l}\text { Corsini et } \\
\text { al. } 2008[18]\end{array}$ & $\begin{array}{l}\text { Stereo light probe } \\
\text { (two reflective } \\
\text { spheres). }\end{array}$ & $\begin{array}{l}\text { The world coordi- } \\
\text { nate origin is co- } \\
\text { incident with the } \\
\text { spheres. }\end{array}$ & $\begin{array}{l}\text { Estimate the light source po- } \\
\text { sitions robustly. }\end{array}$ & $\begin{array}{l}\text { Acquiring light sources and } \\
\text { scene geometry simultane- } \\
\text { ously. }\end{array}$ \\
\hline $\begin{array}{l}\text { Banterle et } \\
\text { al. } 2013 \text { [8] }\end{array}$ & $\begin{array}{l}\text { Single HDR light } \\
\text { probe. }\end{array}$ & $\begin{array}{l}\text { Distant lighting, } \\
\text { relighting as } \\
\text { photographs. }\end{array}$ & $\begin{array}{l}\text { A plausible reconstruction } \\
\text { of the local illumination en- } \\
\text { vironment. }\end{array}$ & $\begin{array}{l}\text { The accuracy of modeling } \\
\text { the scene based on the pos- } \\
\text { sible primitives. Occluded } \\
\text { geometry. }\end{array}$ \\
\hline \multicolumn{5}{|c|}{ REGISTRATION AND ESTIMATION OF LIGHTING } \\
\hline \multicolumn{5}{|c|}{ Explicit Geometric Registration } \\
\hline $\begin{array}{l}\text { Debevec et } \\
\text { al. } 2004[21]\end{array}$ & $\begin{array}{l}\text { HDR light probes. } \\
\text { Reflectance prop- } \\
\text { erties are recon- } \\
\text { structed }\end{array}$ & $\begin{array}{l}\text { Isotropic reflection } \\
\text { surfaces where the } \\
\text { light source must } \\
\text { only move within a } \\
\text { single plane of inci- } \\
\text { dence. }\end{array}$ & $\begin{array}{l}\text { Obtain a geometric model } \\
\text { with illumination rendering } \\
\text { consistent from real pho- } \\
\text { tographs and reflectance } \\
\text { properties. }\end{array}$ & $\begin{array}{l}\text { Surfaces with specular re- } \\
\text { flectance are not featured. }\end{array}$ \\
\hline
\end{tabular}


Table 1. comparison of the overviewed methods from the selected papers that we covered forcing on four criteria: required input, assumption, outcome, and drawback.

\begin{tabular}{|c|c|c|c|c|}
\hline Method & Required Input & Assumptions & Outcome & Drawback \\
\hline $\begin{array}{l}\text { Meilland et } \\
\text { al. } 2013 \text { [75] }\end{array}$ & LDR RGB-D. & $\begin{array}{l}\text { The camera re- } \\
\text { sponse function } \\
(\mathrm{CRF}) \text { is a simple } \\
\text { non-linear with the } \\
\text { auto shutter. }\end{array}$ & $\begin{array}{ll}\text { Dense } \quad 3 \mathrm{D} & \mathrm{HDR} \\
\text { environment-model } & \\
\text { estimation. } & \end{array}$ & $\begin{array}{l}\text { Complex to calibrate au- } \\
\text { tomatic shutter variations, } \\
\text { only consider static objects, } \\
\text { flickering. }\end{array}$ \\
\hline \multicolumn{5}{|c|}{ Photometric Registration From Implicit Geometry } \\
\hline $\begin{array}{l}\text { Nishino et } \\
\text { al. } 2004 \text { [79] }\end{array}$ & $\begin{array}{l}\text { Environment map } \\
\text { using the human } \\
\text { eye as a probe. }\end{array}$ & $\begin{array}{l}\text { The eccentricity } \\
\text { and curvature ra- } \\
\text { dius at the apex, the } \\
\text { same intensity for } \\
\text { the entire image. }\end{array}$ & $\begin{array}{l}\text { Estimate the illumination } \\
\text { of the scene from imaging } \\
\text { corneal system and relight- } \\
\text { ing faces. }\end{array}$ & $\begin{array}{l}\text { Limited dynamic range, the } \\
\text { limited extent of reflections. }\end{array}$ \\
\hline $\begin{array}{l}\text { Knorr } \\
\text { and Kurz } \\
2014[55]\end{array}$ & $\begin{array}{l}\text { A single image of a } \\
\text { human face. }\end{array}$ & $\begin{array}{l}\text { A distant light, } \\
\text { close frontal head } \\
\text { poses needed. }\end{array}$ & $\begin{array}{l}\text { Estimate the real scene } \\
\text { lighting condition in real- } \\
\text { time. }\end{array}$ & $\begin{array}{l}\text { Only focus on the frontal } \\
\text { pose, the approximation of } \\
\text { radiance transfer function } \\
\text { (RTF) is coarse. }\end{array}$ \\
\hline \multicolumn{5}{|c|}{ Photometric Registration From A Specular } \\
\hline $\begin{array}{l}\text { Jachnik et } \\
\text { al. } 2012[41]\end{array}$ & $\begin{array}{l}\text { Live Image of Spec- } \\
\text { ular surface. }\end{array}$ & $\begin{array}{l}\text { Distant light, con- } \\
\text { stant reflection, } \\
\text { Consistent spec- } \\
\text { ular component } \\
\text { through the whole } \\
\text { surface, The sur- } \\
\text { face radiance is } \\
\text { proportional to the } \\
\text { irradiance. }\end{array}$ & $\begin{array}{l}\text { Dense illumination infor- } \\
\text { mation in real-time from } \\
\text { the surface light field. }\end{array}$ & $\begin{array}{l}\text { Limited dynamic range by } \\
\text { the camera. }\end{array}$ \\
\hline \multicolumn{5}{|c|}{ Photometric Registration From Diffuse Reflections } \\
\hline $\begin{array}{l}\text { Gruber et al. } \\
2012 \text { [35] }\end{array}$ & $\begin{array}{l}\text { RGB-D sensor color } \\
\text { Image and depth } \\
\text { map. }\end{array}$ & $\begin{array}{l}\text { Known scene ge- } \\
\text { ometry, diffuse sur- } \\
\text { faces (Lambertian } \\
\text { reflectance model), } \\
\text { the light color is } \\
\text { white and distant. }\end{array}$ & $\begin{array}{l}\text { Estimate light from the ob- } \\
\text { served reconstructed model } \\
\text { using } \mathrm{SH} \text {. }\end{array}$ & $\begin{array}{l}\text { Slow dynamic reconstruc- } \\
\text { tion, static camera, visual ar- } \\
\text { tifacts such as aliasing. The } \\
\text { quality depends on surface } \\
\text { normal vectors, only diffuse } \\
\text { shadow, and lighting. }\end{array}$ \\
\hline
\end{tabular}


Table 1. comparison of the overviewed methods from the selected papers that we covered forcing on four criteria: required input, assumption, outcome, and drawback.

\begin{tabular}{|c|c|c|c|c|}
\hline Method & Required Input & Assumptions & Outcome & Drawback \\
\hline $\begin{array}{l}\text { Gruber et al. } \\
2015[36]\end{array}$ & $\begin{array}{l}\text { RGB-D sensor color } \\
\text { Image and depth } \\
\text { map. }\end{array}$ & $\begin{array}{l}\text { The light is distant } \\
\text { and white. Estimate } \\
\text { light with visual co- } \\
\text { herence in dynamic } \\
\text { scenes. }\end{array}$ & $\begin{array}{l}\text { Restrict to diffuse light } \\
\text { transport and materials. }\end{array}$ & $\begin{array}{l}\text { Geometry reconstruction } \\
\text { quality and depth range are } \\
\text { based on the sensor, visual } \\
\text { artifacts. }\end{array}$ \\
\hline $\begin{array}{l}\text { Boom et al. } \\
2017 \text { [12] }\end{array}$ & $\begin{array}{l}\text { RGB-D sensor color } \\
\text { Image and depth } \\
\text { map. }\end{array}$ & $\begin{array}{l}\text { Diffuse surfaces } \\
\text { (Lambertian re- } \\
\text { flectance model). }\end{array}$ & $\begin{array}{l}\text { Estimate a point light posi- } \\
\text { tion in the recorded scene. }\end{array}$ & $\begin{array}{l}\text { Estimation of a Single point } \\
\text { light source only, limited } \\
\text { cast shadow. }\end{array}$ \\
\hline \multicolumn{5}{|c|}{ Photometric Registration } \\
\hline $\begin{array}{l}\text { Haller et al. } \\
2003[37]\end{array}$ & $\begin{array}{l}\text { Image and depth } \\
\text { information of the } \\
\text { real objects. }\end{array}$ & $\begin{array}{l}\text { The light does not } \\
\text { fall directly on the } \\
\text { non-rigid objects, } \\
\text { distant scene. }\end{array}$ & $\begin{array}{l}\text { Calculate the silhouettes to } \\
\text { extract the shadow volume } \\
\text { in real-time and estimate } \\
\text { the light source. }\end{array}$ & $\begin{array}{l}\text { Shadow volume limited to a } \\
\text { certain radius (distance be- } \\
\text { tween two objects), limited } \\
\text { stencil volume size, and one } \\
\text { light source only. }\end{array}$ \\
\hline $\begin{array}{l}\text { Shi } \\
2017[97]\end{array}$ & $\begin{array}{l}\text { Two HDR video } \\
\text { one for environ- } \\
\text { ment map and } \\
\text { other for input. }\end{array}$ & $\begin{array}{l}\text { Specular compo- } \\
\text { nent not significant } \\
\text { for the light source. }\end{array}$ & $\begin{array}{l}\text { realistic reflection effects } \\
\text { based on the physical light- } \\
\text { ing conditions It worked } \\
\text { on limited parameter adjust- } \\
\text { ments and BRDF models } \\
\text { based on user edit. }\end{array}$ & Limited materials. \\
\hline \multicolumn{5}{|c|}{ Photometric Registration from Images } \\
\hline $\begin{array}{l}\text { Marschner } \\
1999- \\
1998 \quad[71, \\
72]\end{array}$ & $\begin{array}{l}\text { A series of pho- } \\
\text { tographs and con- } \\
\text { vexly curved sam- } \\
\text { ples with homoge- } \\
\text { neous BRDF }\end{array}$ & Stable light source & $\begin{array}{l}\text { high resolution and accu- } \\
\text { racy of surface material on a } \\
\text { large scale illumination and } \\
\text { reflection directions with- } \\
\text { out any special equipment. }\end{array}$ & $\begin{array}{l}\text { Only work with flat sam- } \\
\text { ples. }\end{array}$ \\
\hline 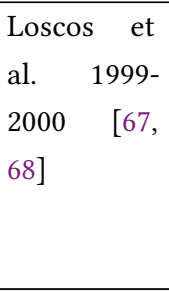 & $\begin{array}{l}\text { A series of pho- } \\
\text { tographs from dif- } \\
\text { ferent several view- } \\
\text { point }\end{array}$ & Surfaces are diffuse. & $\begin{array}{l}\text { Reconstructing real scene } \\
\text { geometry and present } \\
\text { a method to recover re- } \\
\text { flectance and interactive } \\
\text { relighting considering } \\
\text { shadow. }\end{array}$ & $\begin{array}{l}\text { Does not include indirect } \\
\text { lighting calculations. }\end{array}$ \\
\hline $\begin{array}{l}\text { Poulin et al. } \\
1998 \text { [84] }\end{array}$ & Set of photographs & $\begin{array}{l}\text { Arbitrary camera } \\
\text { parameters. }\end{array}$ & $\begin{array}{l}\text { 3D geometry reconstruc- } \\
\text { tion and texture extraction. }\end{array}$ & $\begin{array}{l}\text { User interference, not an au- } \\
\text { tomated system. }\end{array}$ \\
\hline
\end{tabular}


Table 1. comparison of the overviewed methods from the selected papers that we covered forcing on four criteria: required input, assumption, outcome, and drawback.

\begin{tabular}{|c|c|c|c|c|}
\hline Method & Required Input & Assumptions & Outcome & Drawback \\
\hline $\begin{array}{l}\text { Mandl et al. } \\
2017 \text { [70] }\end{array}$ & $\begin{array}{l}\text { Pure synthetic im- } \\
\text { ages which then ap- } \\
\text { plied to real image } \\
\text { data-set. }\end{array}$ & $\begin{array}{l}\text { Known object in the } \\
\text { scene }\end{array}$ & $\begin{array}{l}\text { Train convolution neural } \\
\text { network }(\mathrm{CNN}) \text { for high- } \\
\text { quality illumination estima- } \\
\text { tion }\end{array}$ & $\begin{array}{l}\text { Limited dataset, large CNN } \\
\text { instances, not an automated } \\
\text { system for object recogni- } \\
\text { tion. }\end{array}$ \\
\hline $\begin{array}{l}\text { Weber et al. } \\
2018 \text { [107] }\end{array}$ & $\begin{array}{l}\text { single image, } \\
\text { a database of } \\
\text { environment maps }\end{array}$ & Known 3D object & $\begin{array}{l}\text { Developing a deep learning } \\
\text { method to estimate indoor } \\
\text { lighting. }\end{array}$ & $\begin{array}{l}\text { Must re-train different il- } \\
\text { lumination prediction net- } \\
\text { works for other new ob- } \\
\text { jects and material proper- } \\
\text { ties. Must estimate geome- } \\
\text { try and material properties } \\
\text { to train the neural network. }\end{array}$ \\
\hline $\begin{array}{l}\text { Gardner et } \\
\text { al. } 2017 \text { [28] }\end{array}$ & $\begin{array}{l}\text { a single and lim- } \\
\text { ited field of view im- } \\
\text { age, LDR environ- } \\
\text { ment map dataset }\end{array}$ & $\begin{array}{l}\text { Spherical Scene, ig- } \\
\text { nore occlusion }\end{array}$ & $\begin{array}{l}\text { A lighting classifier that } \\
\text { is robust and annotate the } \\
\text { light location automatically } \\
\text { and train a deep neural } \\
\text { network for light location } \\
\text { and intensity prediction in } \\
\text { a scene. }\end{array}$ & $\begin{array}{l}\text { The warping operator can- } \\
\text { not model occlusions. The } \\
\text { method failed on images } \\
\text { that have ambiguous pho- } \\
\text { tometric or geometric cues. } \\
\text { The light size could be de- } \\
\text { tected as smaller than it } \\
\text { seems. }\end{array}$ \\
\hline \multicolumn{5}{|c|}{ Photometric Registration For Outdoor Scenes } \\
\hline $\begin{array}{l}\text { Lalonde et } \\
\text { al. 2009 [62] }\end{array}$ & $\begin{array}{l}\text { single outdoor im- } \\
\text { age }\end{array}$ & $\begin{array}{l}\text { The surfaces albe- } \\
\text { dos are known, the } \\
\text { sun visibility inde- } \\
\text { pendent from its po- } \\
\text { sition. }\end{array}$ & $\begin{array}{l}\text { Calculate the probability } \\
\text { distribution over the posi- } \\
\text { tion of the sunlight and } \\
\text { its visibility to estimate the } \\
\text { scene illumination condi- } \\
\text { tions. }\end{array}$ & $\begin{array}{l}\text { Some resulting estimations } \\
\text { are weak. Several assump- } \\
\text { tions to reduce the problem } \\
\text { complexity which could not } \\
\text { be true all the time. }\end{array}$ \\
\hline $\begin{array}{l}\text { Liu et al. } \\
2015[65]\end{array}$ & $\begin{array}{l}\text { Single outdoor im- } \\
\text { age. }\end{array}$ & $\begin{array}{l}\text { The shadow casts } \\
\text { on planar Lamber- } \\
\text { tian surfaces, object } \\
\text { position at world } \\
\text { coordinate origin. }\end{array}$ & $\begin{array}{l}\text { Estimate sunlight direction } \\
\text { using the shadow cast on } \\
\text { object modeling and recog- } \\
\text { nition. }\end{array}$ & $\begin{array}{l}\text { Limited range of the view- } \\
\text { point, object bounding box } \\
\text { with inaccuracy detection } \\
\text { could lead to error in sun- } \\
\text { light direction estimation. }\end{array}$ \\
\hline
\end{tabular}


Table 1. comparison of the overviewed methods from the selected papers that we covered forcing on four criteria: required input, assumption, outcome, and drawback.

\begin{tabular}{|c|c|c|c|c|}
\hline Method & Required Input & Assumptions & Outcome & Drawback \\
\hline $\begin{array}{l}\text { Barreira et } \\
\text { al. } 2018[10]\end{array}$ & $\begin{array}{l}\text { GPS location, } \\
\text { weather API for sky } \\
\text { condition, ALS of } \\
\text { the mobile device } \\
\text { for illuminance } \\
\text { measure. }\end{array}$ & Outdoor scenes. & $\begin{array}{l}\text { Estimate the illumination } \\
\text { condition then reconstruct } \\
\text { the sun position and di- } \\
\text { rection, detect dynamic } \\
\text { shadow. }\end{array}$ & $\begin{array}{l}\text { The object and mobile de- } \\
\text { vice should be in the same } \\
\text { location. The object not } \\
\text { halfway in shadow. Nonlin- } \\
\text { ear color correction, the sys- } \\
\text { tem only works in outdoor. }\end{array}$ \\
\hline \multicolumn{5}{|c|}{ COMPOSITION AND GLOBAL ILLUMINATION } \\
\hline \multicolumn{5}{|c|}{ Shadows In Common Illumination } \\
\hline $\begin{array}{l}\text { Ritschel } \\
2011[88]\end{array}$ & $\begin{array}{l}\text { High quality for } \\
\text { Imperfect Shadow } \\
\text { Maps (ISM). }\end{array}$ & $\begin{array}{l}\text { Known geometric } \\
\text { model of the scene } \\
\text { and light informa- } \\
\text { tion. }\end{array}$ & $\begin{array}{l}\text { Compute interactive Indi- } \\
\text { rect illumination in dy- } \\
\text { namic scenes Some spatial } \\
\text { details could be lost. }\end{array}$ & The light should be smooth. \\
\hline $\begin{array}{l}\text { Everitt and } \\
\text { Kilgard } \\
2003[22]\end{array}$ & $\begin{array}{l}\text { Triangles Models } \\
\text { for occluding } \\
\text { objects. }\end{array}$ & $\begin{array}{l}\text { Ideal point light } \\
\text { source. Detect the } \\
\text { shadow area in the } \\
\text { scene. }\end{array}$ & $\begin{array}{l}\text { Incorrect shadow based on } \\
\text { shadow depth count of the } \\
\text { stencil value. }\end{array}$ & $\begin{array}{l}\text { Ineffective use of the two } \\
\text { passes render. }\end{array}$ \\
\hline \multicolumn{5}{|c|}{ Diffuse Global Illumination } \\
\hline $\begin{array}{l}\text { Rohmer et } \\
\text { al. } 2015 \text { [89] }\end{array}$ & $\begin{array}{l}\text { Several HDR fish- } \\
\text { eye video cameras. }\end{array}$ & $\begin{array}{l}\text { The low frequency } \\
\text { for the remaining } \\
\text { illumination of the } \\
\text { indirect radiance at- } \\
\text { las. }\end{array}$ & $\begin{array}{l}\text { Consistent simulation of } \\
\text { the illumination effects } \\
\text { adapting to temporal } \\
\text { deviations. }\end{array}$ & $\begin{array}{l}\text { Required external tracking } \\
\text { and was designed for dif- } \\
\text { fused objects more than } \\
\text { other materials. }\end{array}$ \\
\hline $\begin{array}{l}\text { Franke } \\
2013[26]\end{array}$ & $\begin{array}{l}\text { Image and depth } \\
\text { map from the RGB- } \\
\text { D sensor. }\end{array}$ & $\begin{array}{l}\text { Known scene geom- } \\
\text { etry. }\end{array}$ & $\begin{array}{l}\text { Visual coherence and re- } \\
\text { lighting method for realistic } \\
\text { indirect reflection between } \\
\text { real and virtual objects. }\end{array}$ & $\begin{array}{l}\text { Illumination bleeding and } \\
\text { anti-radiance cause by } \\
\text { some artifacts and the high } \\
\text { cost of the procedure. }\end{array}$ \\
\hline \multicolumn{5}{|c|}{ Specular Global Illumination } \\
\hline $\begin{array}{l}\text { Knecht } \\
\text { et al., } \\
2010[54]\end{array}$ & $\begin{array}{l}\text { LDR video using } \\
\text { the fish-eye camera. }\end{array}$ & $\begin{array}{l}\text { Available geometric } \\
\text { model of the real } \\
\text { scene with known } \\
\text { martial properties }\end{array}$ & $\begin{array}{l}\text { Simulate the direct incident } \\
\text { light and track the camera } \\
\text { and lamp position. }\end{array}$ & $\begin{array}{l}\text { Double shadowing and in- } \\
\text { consistent coloring in some } \\
\text { cases. }\end{array}$ \\
\hline $\begin{array}{l}\text { Kan and } \\
\text { Kaufmann } \\
2012[48]\end{array}$ & $\begin{array}{l}3 \mathrm{D} \text { scene data, } \\
\text { video image, en- } \\
\text { vironment image } \\
\text { fish-eye. }\end{array}$ & Static objects. & $\begin{array}{l}\text { High-quality specular ef- } \\
\text { fects with the depth of field } \\
\text { effect and anti-aliasing. }\end{array}$ & $\begin{array}{l}\text { Color bleeding and dif- } \\
\text { fuse indirect lighting not } \\
\text { featured, accurate camera } \\
\text { tracking. }\end{array}$ \\
\hline
\end{tabular}


Real-Time Illumination and Visual Coherence for Photorealistic Augmented/Mixed Reality

\section{REFERENCES}

[1] Nor Azlina Ab Aziz, Kamarulzaman Ab Aziz, Avijit Paul, Anuar Mohd Yusof, and Noor Shuhailie Mohamed Noor. 2012. Providing augmented reality based education for students with attention deficit hyperactive disorder via cloud computing: Its advantages. In 2012 14th International Conference on Advanced Communication Technology (ICACT). 577-581.

[2] Kusuma Agusanto, Li Li, Zhu Chuangui, and Ng Wan Sing. 2003. Photorealistic rendering for augmented reality using environment illumination. In The Second IEEE and ACM International Symposium on Mixed and Augmented Reality, 2003. Proceedings. 208-216. https://doi.org/10.1109/ISMAR. 2003.1240704

[3] A'aeshah Alhakamy and Mihran Tuceryan. 2019. AR360: Dynamic Illumination for Augmented Reality with Real-Time Interaction. In 2019 IEEE 2nd International Conference on Information and Computer Technologies (ICICT). 170-174. https://doi.org/10.1109/INFOCT.2019.8710982

[4] A'aeshah Alhakamy and Mihran Tuceryan. 2019. CubeMap360: Interactive Global Illumination for Augmented Reality in Dynamic Environment.. In 2019 SoutheastCon. IEEE, IEEE Press, 1-8. https://doi.org/10.1109/SoutheastCon42311.2019.9020588

[5] A'aeshah Alhakamy and Mihran Tuceryan. 2019. An Empirical Evaluation of the Performance of Real-Time Illumination Approaches: Realistic Scenes in Augmented Reality. In Augmented Reality, Virtual Reality, and Computer Graphics. Springer International Publishing, Cham, $179-195$. https://doi.org/10.1007/978-3-030-25999-0_16

[6] A'aeshah Alhakamy and Mihran Tuceryan. 2019. Polarization-Based Illumination Detection for Coherent Augmented Reality Scene Rendering in Dynamic Environments. In Proceedings of the 36th Computer Graphics International (CGI'19) in cooperation with ACM SIGGRAPH and Eurographics, chapter in (Advances in Computer Graphics). Springer International Publishing, Cham, 3-14. https://doi.org/10.1007/978-3-030-22514-8_1

[7] A'aeshah Alhakamy and Mihran Tuceryan. 2020. Physical Environment Reconstruction Beyond Light Polarization for Coherent Augmented Reality Scene on Mobile Devices. In Transactions on Computational Science XXXVII: Special Issue on Computer Graphics. Springer Berlin Heidelberg, Berlin, Heidelberg, 19-38. https://doi.org/10.1007/978-3-662-61983-4_2

[8] Francesco Banterle, Marco Callieri, Matteo Dellepiane, Massimiliano Corsini, Fabio Pellacini, and Roberto Scopigno. 2013. EnvyDepth: An Interface for Recovering Local Natural Illumination from Environment Maps. Computer Graphics Forum 32, 7, 411-420. https://doi.org/10.1111/cgf.12249 arXiv:https://onlinelibrary.wiley.com/doi/pdf/10.1111/cgf.12249

[9] Yoram Baram. 2004. Closed-loop augmented reality apparatus. US Patent 6,734,834.

[10] João Barreira, Maximino Bessa, Luís Barbosa, and Luís Magalhães. 2018. A Context-Aware Method for Authentically Simulating Outdoors Shadows for Mobile Augmented Reality. IEEE Transactions on Visualization and Computer Graphics 24, 3 (March 2018), 1223-1231. https: //doi.org/10.1109/TVCG.2017.2676777

[11] Jonathan T Barron and Jitendra Malik. 2015. Shape, Illumination, and Reflectance from Shading. IEEE Transactions on Pattern Analysis and Machine Intelligence 37, 8 (Aug 2015), 1670-1687. https://doi.org/10.1109/TPAMI.2014.2377712

[12] Bastiaan J. Boom, Sergio Orts-Escolano, Xin X. Ning, Steven McDonagh, Peter Sandilands, and Robert B. Fisher. 2017. Interactive light source position estimation for augmented reality with an RGB-D camera. Computer Animation and Virtual Worlds 28, 1 (2017), e1686. https://doi.org/10.1002/cav.1686 arXiv:https://onlinelibrary.wiley.com/doi/pdf/10.1002/cav.1686 e1686 cav.1686.

[13] Derek Bradley, Gerhard Roth, and Prosenjit Bose. 2009. Augmented reality on cloth with realistic illumination. Machine Vision and Applications 20, 2 (2009), 85-92. https://doi.org/10.1007/s00138-007-0108-9

[14] David E. Breen, Ross T. Whitaker, Eric Rose, and Mihran Tuceryan. 1996. Interactive Occlusion and Automatic Object Placement for Augmented Reality. Computer Graphics Forum 15, 3, 11-22. https://doi.org/10.1111/1467-8659.1530011 arXiv:https://onlinelibrary.wiley.com/doi/pdf/10.1111/14678659.1530011

[15] Xavier Casas, Gerardo Herrera, Inmaculada Coma, and Marcos Fernãndez. 2012. A kinect-based augmented reality system for individuals with autism spectrum disorders. In Proceedings of the International Conference on Computer Graphics Theory and Applications and International Conference on Information Visualization Theory and Applications - Volume 1: GRAPP, (VISIGRAPP 2012). INSTICC, SciTePress, 440-446. https: //doi.org/10.5220/0003844204400446

[16] Andrew I Comport, Éric Marchand, and François Chaumette. 2003. A real-time tracker for markerless augmented reality. In The Second IEEE and ACM International Symposium on Mixed and Augmented Reality, 2003. Proceedings. 36-45. https://doi.org/10.1109/ISMAR.2003.1240686

[17] Jon Cook, Simon Gibson, Toby Howard, and Roger Hubbold. 2003. Real-Time Photo-Realistic Augmented Reality for Interior Design. In ACM SIGGRAPH 2003 Sketches \& Applications (SIGGRAPH '03). Association for Computing Machinery, New York, NY, USA, 1. https://doi.org/10.1145/ 965400.965427

[18] Massimiliano Corsini, Marco Callieri, and Paolo Cignoni. 2008. Stereo Light Probe. Computer Graphics Forum 27, 2, 291-300. https://doi.org/10. 1111/j.1467-8659.2008.01126.x arXiv:https://onlinelibrary.wiley.com/doi/pdf/10.1111/j.1467-8659.2008.01126.x

[19] Paul Debevec. 2008. Rendering Synthetic Objects into Real Scenes: Bridging Traditional and Image-Based Graphics with Global Illumination and High Dynamic Range Photography. In ACM SIGGRAPH 2008 Classes (SIGGRAPH '08). Association for Computing Machinery, New York, NY, USA, Article Article 32, 10 pages. https://doi.org/10.1145/1401132.1401175

[20] Paul Debevec, Paul Graham, Jay Busch, and Mark Bolas. 2012. A Single-Shot Light Probe. In ACM SIGGRAPH 2012 Talks (SIGGRAPH '12). Association for Computing Machinery, New York, NY, USA, Article Article 10, 1 pages. https://doi.org/10.1145/2343045.2343058

[21] Paul Debevec, Chris Tchou, Andrew Gardner, Tim Hawkins, Charis Poullis, Jessi Stumpfel, Andrew Jones, Nathaniel Yun, Per Einarsson, Therese Lundgren, et al. 2004. Estimating surface reflectance properties of a complex scene under captured natural illumination. 
[22] Cass W. Everitt and Mark J. Kilgard. 2003. Practical and Robust Stenciled Shadow Volumes for Hardware-Accelerated Rendering. CoRR cs.GR/0301002 (2003). http://arxiv.org/abs/cs/0301002

[23] Alain Fournier, Atjeng S Gunawan, and Chris Romanzin. 1993. Common illumination between real and computer generated scenes. In Graphics Interface. CANADIAN INFORMATION PROCESSING SOCIETY, 254-254.

[24] Jan-Michael Frahm, Kevin Koeser, Daniel Grest, and Reinhard Koch. 2005. Markerless augmented reality with light source estimation for direct illumination. In Conference on Visual Media Production CVMP, London. IET, 211-220.

[25] Banterle Francesco, Artusi Alessandro, Debattista Kurt, and Chalmers Alan. 2017. Advanced High Dynamic Range Imaging (2nd edition ed.). A K Peters/CRC Press. 328 pages. https://doi.org/10.1201/9781315119526

[26] Tobias Alexander Franke. 2013. Delta Light Propagation Volumes for mixed reality. In 2013 IEEE International Symposium on Mixed and Augmented Reality (ISMAR). 125-132. https://doi.org/10.1109/ISMAR.2013.6671772

[27] Tobias Alexander Franke. 2014. Delta Voxel Cone Tracing. In 2014 IEEE International Symposium on Mixed and Augmented Reality (ISMAR). 39-44. https://doi.org/10.1109/ISMAR.2014.6948407

[28] Marc-André Gardner, Kalyan Sunkavalli, Ersin Yumer, Xiaohui Shen, Emiliano Gambaretto, Christian Gagné, and Jean-François Lalonde. 2017. Learning to Predict Indoor Illumination from a Single Image. ACM Trans. Graph. 36, 6, Article 176 (Nov. 2017), 14 pages. https://doi.org/10.1145/ 3130800.3130891

[29] Simon Gibson, Jon Cook, Toby Howard, and Roger Hubbold. 2003. Rapid Shadow Generation in Real-World Lighting Environments. In Proceedings of the 14th Eurographics Workshop on Rendering (EGRW'03). Eurographics Association, Goslar, DEU, 219-229.

[30] Simon Gibson and Alan Murta. 2000. Interactive Rendering with Real-World Illumination. In Rendering Techniques 2000, Bernard Péroche and Holly Rushmeier (Eds.). Springer Vienna, Vienna, 365-376. https://doi.org/10.1007/978-3-7091-6303-0_33

[31] Robin Green. 2003. Spherical harmonic lighting: The gritty details. In Archives of the Game Developers Conference, Vol. 56.4

[32] Thorsten Grosch. 2005. Differential Photon Mapping-Consistent Augmentation of Photographs with Correction of all Light Paths.. In Eurographics (Short Presentations). 53-56

[33] Thorsten Grosch. 2005. PanoAR: Interactive augmentation of omnidirectional images with consistent lighting. Mirage 2005, Computer Vision/Computer Graphics Collaboration Techniques and Applications (2005), 25-34.

[34] Thorsten Grosch, Tobias Eble, and Stefan Mueller. 2007. Consistent Interactive Augmentation of Live Camera Images with Correct Near-Field Illumination. In Proceedings of the 2007 ACM Symposium on Virtual Reality Software and Technology (VRST '07). Association for Computing Machinery, New York, NY, USA, 125-132. https://doi.org/10.1145/1315184.1315207

[35] Lukas Gruber, Thomas Richter-Trummer, and Dieter Schmalstieg. 2012. Real-time photometric registration from arbitrary geometry. In 2012 IEEE International Symposium on Mixed and Augmented Reality (ISMAR). 119-128. https://doi.org/10.1109/ISMAR.2012.6402548

[36] Lukas Gruber, Jonathan Ventura, and Dieter Schmalstieg. 2015. Image-space illumination for augmented reality in dynamic environments. In 2015 IEEE Virtual Reality (VR). 127-134. https://doi.org/10.1109/VR.2015.7223334

[37] Michael Haller, Stephan Drab, and Werner Hartmann. 2003. A Real-Time Shadow Approach for an Augmented Reality Application Using Shadow Volumes. In Proceedings of the ACM Symposium on Virtual Reality Software and Technology (VRST '03). Association for Computing Machinery, New York, NY, USA, 56-65. https://doi.org/10.1145/1008653.1008665

[38] Jassim Happa, Thomas Bashford-Rogers, Kurt Debattista, and Alan Chalmers. 2011. Rendering Interior Cultural Heritage Scenes Using Image-based Shooting.. In Eurographics (Areas Papers). 49-56.

[39] Vlastimil Havran, Miloslaw Smyk, Grzegorz Krawczyk, Karol Myszkowski, and Hans-Peter Seidel. 2005. Importance Sampling for Video Environment Maps. In ACM SIGGRAPH 2005 Sketches (SIGGRAPH '05). Association for Computing Machinery, New York, NY, USA, 109-es. https://doi.org/10.1145/1187112.1187243

[40] Shahram Izadi, David Kim, Otmar Hilliges, David Molyneaux, Richard Newcombe, Pushmeet Kohli, Jamie Shotton, Steve Hodges, Dustin Freeman, Andrew Davison, and et al. 2011. KinectFusion: Real-Time 3D Reconstruction and Interaction Using a Moving Depth Camera. In Proceedings of the 24th Annual ACM Symposium on User Interface Software and Technology (UIST '11). Association for Computing Machinery, New York, NY, USA, 559-568. https://doi.org/10.1145/2047196.2047270

[41] Jan Jachnik, Richard A Newcombe, and Andrew J Davison. 2012. Real-time surface light-field capture for augmentation of planar specular surfaces. In 2012 IEEE International Symposium on Mixed and Augmented Reality (ISMAR). 91-97. https://doi.org/10.1109/ISMAR.2012.6402544

[42] Katrien Jacobs and Céline Loscos. 2006. Classification of Illumination Methods for Mixed Reality. Computer Graphics Forum 25, 1, 29-51. https://doi.org/10.1111/j.1467-8659.2006.00816.x arXiv:https://onlinelibrary.wiley.com/doi/pdf/10.1111/j.1467-8659.2006.00816.x

[43] Henrik Wann Jensen and Niels Jørgen Christensen. 1995. Photon maps in bidirectional Monte Carlo ray tracing of complex objects. Computers \& Graphics 19, 2 (1995), 215 - 224. https://doi.org/10.1016/0097-8493(94)00145-O

[44] Salma Jiddi, Philippe Robert, and Eric Marchand. 2016. Reflectance and illumination estimation for realistic augmentations of real scenes. In 2016 IEEE International Symposium on Mixed and Augmented Reality (ISMAR-Adjunct). IEEE, 244-249.

[45] M Carmen Juan, Mariano Alcaniz, Carlos Monserrat, Cristina Botella, Rosa María Baños, and Belen Guerrero. 2005. Using augmented reality to treat phobias. IEEE Computer Graphics and Applications 25, 6 (Nov 2005), 31-37. https://doi.org/10.1109/MCG.2005.143

[46] James T. Kajiya. 1986. The Rendering Equation. In Proceedings of the 13th Annual Conference on Computer Graphics and Interactive Techniques (SIGGRAPH '86). Association for Computing Machinery, New York, NY, USA, 143-150. https://doi.org/10.1145/15922.15902 
[47] Divya Kamboj and Wankui Liu. 2014. Improved Variance Cut Algorithm for Light Source Estimation in Augmented Reality. International fournal of Computer Applications 85, 19 (2014).

[48] Peter Kán and Hannes Kaufmann. 2012. High-quality reflections, refractions, and caustics in Augmented Reality and their contribution to visual coherence. In 2012 IEEE International Symposium on Mixed and Augmented Reality (ISMAR). 99-108. https://doi.org/10.1109/ISMAR.2012.6402546

[49] Peter Kán and Hannes Kaufmann. 2013. Differential Irradiance Caching for fast high-quality light transport between virtual and real worlds. In 2013 IEEE International Symposium on Mixed and Augmented Reality (ISMAR). 133-141. https://doi.org/10.1109/ISMAR.2013.6671773

[50] Masayuki Kanbara and Naokazu Yokoya. 2004. Real-time estimation of light source environment for photorealistic augmented reality. In Proceedings of the 17th International Conference on Pattern Recognition, 2004. (ICPR'04), Vol. 2. IEEE Cambridge, UK, 911-914. https://doi.org/10.1109/ICPR.2004. 1334407

[51] Kevin Karsch, Varsha Hedau, David Forsyth, and Derek Hoiem. 2011. Rendering Synthetic Objects into Legacy Photographs. In Proceedings of the 2011 SIGGRAPH Asia Conference (SA '11). Association for Computing Machinery, New York, NY, USA, Article Article 157, 12 pages. https://doi.org/10.1145/2024156.2024191

[52] Kevin Karsch, Kalyan Sunkavalli, Sunil Hadap, Nathan Carr, Hailin Jin, Rafael Fonte, Michael Sittig, and David Forsyth. 2014. Automatic Scene Inference for 3D Object Compositing. ACM Trans. Graph. 33, 3, Article Article 32 (June 2014), 15 pages. https://doi.org/10.1145/2602146

[53] Jan Kautz, John Snyder, and Peter-Pike J Sloan. 2002. Fast Arbitrary BRDF Shading for Low-Frequency Lighting Using Spherical Harmonics. Rendering Techniques 2, 291-296 (2002), 1.

[54] Martin Knecht, Christoph Traxler, Oliver Mattausch, Oliver Mattausch, Purgathofer Werner, and Wimmer Michael. 2010. Differential Instant Radiosity for mixed reality. In 2010 IEEE International Symposium on Mixed and Augmented Reality. 99-107. https://doi.org/10.1109/ISMAR.2010. 5643556

[55] Sebastian B Knorr and Daniel Kurz. 2014. Real-time illumination estimation from faces for coherent rendering. In 2014 IEEE International Symposium on Mixed and Augmented Reality (ISMAR). 113-122. https://doi.org/10.1109/ISMAR.2014.6948416

[56] Hoshang Kolivand and Mohd Shahrizal Sunar. 2013. Survey of Shadow Volume Algorithms in Computer Graphics. IETE Technical Review 30, 1 (2013), 38-46. https://doi.org/10.4103/0256-4602.107338 arXiv:https://www.tandfonline.com/doi/pdf/10.4103/0256-4602.107338

[57] Hoshang Kolivand and Mohd Shahrizal Sunar. 2014. Realistic real-time outdoor rendering in augmented reality. PloS one 9, 9 (2014), e108334. https://doi.org/doi:10.1371/journal.pone.0108334

[58] Hoshang Kolivand, Mohd Shahrizal Sunar, Samira Y Kakh, Riyadh Al-Rousan, and Ismahafezi Ismail. 2018. Photorealistic rendering: a survey on evaluation. Multimedia Tools and Applications 77, 19 (2018), 25983-26008. https://doi.org/10.1007/s11042-018-5834-7

[59] Hoshang Kolivand, Mohd Shahrizal Sunar, Tanzila Saba, and Hatem Ali. 2019. ReLiShaft: realistic real-time light shaft generation taking sky illumination into account. Multimedia Tools and Applications 78, 5 (2019), 6073-6092. https://doi.org/10.1007/s11042-018-6296-7

[60] Matthias Korn, Maik Stange, Andreas von Arb, Lisa Blum, Michael Kreil, Kathrin-Jennifer Kunze, Jens Anhenn, Timo Wallrath, and Thorsten Grosch. 2008. Interactive Augmentation of Live Images using a HDR Stereo Camera. FVRB - fournal of Virtual Reality and Broadcasting Vol. 4, 12 (2008). https://doi.org/10.20385/1860-2037/4.2007.12

[61] Joel Kronander, Francesco Banterle, Andrew Gardner, Ehsan Miandji, and Jonas Unger. 2015. Photorealistic rendering of mixed reality scenes. Computer Graphics Forum 34, 2, 643-665. https://doi.org/10.1111/cgf.12591 arXiv:https://onlinelibrary.wiley.com/doi/pdf/10.1111/cgf.12591

[62] Jean-François Lalonde, Alexei A Efros, and Srinivasa G Narasimhan. 2009. Estimating natural illumination from a single outdoor image. In 2009 IEEE 12th International Conference on Computer Vision. 183-190. https://doi.org/10.1109/ICCV.2009.5459163

[63] Philipp Lensing and Wolfgang Broll. 2012. Instant indirect illumination for dynamic mixed reality scenes. In 2012 IEEE International Symposium on Mixed and Augmented Reality (ISMAR). 109-118. https://doi.org/10.1109/ISMAR.2012.6402547

[64] Philipp Lensing and Wolfgang Broll. 2013. LightSkin: Real-time global illumination for virtual and mixed reality. In Proceedings of the 5th foint Virtual Reality Conference. Eurographics Association, 17-24.

[65] Yang Liu, Theo Gevers, and Xueqing Li. 2015. Estimation of Sunlight Direction Using 3D Object Models. IEEE Transactions on Image Processing 24, 3 (March 2015), 932-942. https://doi.org/10.1109/TIP.2014.2378032

[66] Yanli Liu and Xavier Granier. 2012. Online Tracking of Outdoor Lighting Variations for Augmented Reality with Moving Cameras. IEEE Transactions on Visualization and Computer Graphics 18, 4 (April 2012), 573-580. https://doi.org/10.1109/TVCG.2012.53

[67] Céline Loscos, George Drettakis, and Luc Robert. 2000. Interactive virtual relighting of real scenes. IEEE Transactions on Visualization and Computer Graphics 6, 4 (Oct 2000), 289-305. https://doi.org/10.1109/2945.895874

[68] Céline Loscos, Marie-Claude Frasson, George Drettakis, Bruce Walter, Xavier Granier, and Pierre Poulin. 1999. Interactive Virtual Relighting and Remodeling of Real Scenes. In Rendering Techniques' 99, Dani Lischinski and Greg Ward Larson (Eds.). Springer Vienna, Vienna, 329-340.

[69] Claus B Madsen and Michael Nielsen. 2008. Towards probe-less augmented reality. A Position Paper, Computer Vision and Media Technology Lab, Aalborg University, Aalborg, Denmark (2008).

[70] David Mandl, Kwang Moo Yi, Peter Mohr, Peter M Roth, Pascal Fua, Vincent Lepetit, Dieter Schmalstieg, and Denis Kalkofen. 2017. Learning Lightprobes for Mixed Reality Illumination. In 2017 IEEE International Symposium on Mixed and Augmented Reality (ISMAR). 82-89. https: //doi.org/10.1109/ISMAR.2017.25

[71] Stephen Robert Marschner and Donald P Greenberg. 1998. Inverse rendering for computer graphics. Citeseer.

[72] Stephen R. Marschner, Stephen H. Westin, Eric P. F. Lafortune, Kenneth E. Torrance, and Donald P. Greenberg. 1999. Image-Based BRDF Measurement Including Human Skin. In Rendering Techniques' 99, Dani Lischinski and Greg Ward Larson (Eds.). Springer Vienna, Vienna, 131-144. 
[73] Vincent Masselus, Pieter Peers, Philip Dutré, and Yves D. Willems. 2003. Relighting with 4D Incident Light Fields. ACM Trans. Graph. Vol. 22, 3 , 613-620. https://doi.org/10.1145/882262.882315

[74] Soham Uday Mehta, Kihwan Kim, Dawid Pajak, Kari Pulli, Jan Kautz, and Ravi Ramamoorthi. 2015. Filtering environment illumination for interactive physically-based rendering in mixed reality. In Eurographics Symposium on Rendering, Vol. 1.

[75] Maxime Meilland, Christian Barat, and Andrew Comport. 2013. 3D High Dynamic Range dense visual SLAM and its application to real-time object re-lighting. In 2013 IEEE International Symposium on Mixed and Augmented Reality (ISMAR). 143-152. https://doi.org/10.1109/ISMAR.2013.6671774

[76] Rafael Monroy, Matis Hudon, and Aljosa Smolic. 2018. Dynamic Environment Mapping for Augmented Reality Applications on Mobile Devices. In Vision, Modeling \& Visualization, VMV 2018, Stuttgart, Germany, October 10-12, 2018. 21-28. https://doi.org/10.2312/vmv.20181249

[77] Alexandre Morgand, Mohamed Tamaazousti, and Adrien Bartoli. 2018. A Geometric Model for Specularity Prediction on Planar Surfaces with Multiple Light Sources. IEEE Transactions on Visualization and Computer Graphics 24, 5 (May 2018), 1691-1704. https://doi.org/10.1109/TVCG. 2017.2677445

[78] Brian Mount, Stephen Latta, Daniel McCulloch, Kevin Geisner, Jason Scott, Jonathan Steed, Arthur Tomlin, and Mark Mihelich. 2013. Augmented reality with realistic occlusion. US Patent App. 13/309,372.

[79] Ko Nishino and Shree K. Nayar. 2004. Eyes for Relighting. ACM Trans. Graph. Vol. 23, 3 (Aug. 2004), 704-711. https://doi.org/10.1145/1015706.1015783

[80] Derek Nowrouzezahrai, Stefan Geiger, Kenny Mitchell, Robert Sumner, Wojciech Jarosz, and Markus Gross. 2011. Light factorization for mixed-frequency shadows in augmented reality. In 2011 10th IEEE International Symposium on Mixed and Augmented Reality. 173-179. https: //doi.org/10.1109/ISMAR.2011.6092384

[81] Steven G. Parker, James Bigler, Andreas Dietrich, Heiko Friedrich, Jared Hoberock, David Luebke, David McAllister, Morgan McGuire, Keith Morley, Austin Robison, and et al. 2010. OptiX: A General Purpose Ray Tracing Engine. ACM Trans. Graph. 29, 4, Article Article 66, 13 pages. https://doi.org/10.1145/1778765.1778803

[82] Gustavo Patow and Xavier Pueyo. 2003. A Survey of Inverse Rendering Problems. Computer Graphics Forum 22, 4, 663-687. https://doi.org/10 1111/j.1467-8659.2003.00716.x arXiv:https://onlinelibrary.wiley.com/doi/pdf/10.1111/j.1467-8659.2003.00716.x

[83] Matt Pharr, Wenzel Jakob, and Greg Humphreys. 2016. Physically based rendering: From theory to implementation. Morgan Kaufmann.

[84] Pierre Poulin, Mathieu Ouimet, and Marie-Claude Frasson. 1998. Interactively Modeling with Photogrammetry. In Rendering Techniques '98, George Drettakis and Nelson Max (Eds.). Springer Vienna, Vienna, 93-104.

[85] Ravi Ramamoorthi and Pat Hanrahan. 2001. An Efficient Representation for Irradiance Environment Maps. In Proceedings of the 28th Annual Conference on Computer Graphics and Interactive Techniques (SIGGRAPH '01). Association for Computing Machinery, New York, NY, USA, 497-500. https://doi.org/10.1145/383259.383317

[86] Erik Reinhard, Wolfgang Heidrich, Paul Debevec, Sumanta Pattanaik, Greg Ward, and Karol Myszkowski. 2010. High dynamic range imaging: acquisition, display, and image-based lighting. Morgan Kaufmann.

[87] Thomas Richter-Trummer, Denis Kalkofen, Jinwoo Park, and Dieter Schmalstieg. 2016. Instant Mixed Reality Lighting from Casual Scanning. In 2016 IEEE International Symposium on Mixed and Augmented Reality (ISMAR). 27-36. https://doi.org/10.1109/ISMAR.2016.18

[88] Tobias Ritschel, Elmar Eisemann, Inwoo Ha, James D. K. Kim, and Hans-Peter Seidel. 2011. Making Imperfect Shadow Maps View-Adaptive: HighQuality Global Illumination in Large Dynamic Scenes. Computer Graphics Forum 30, 8, 2258-2269. https://doi.org/10.1111/j.1467-8659.2011.01998.x arXiv:https:/onlinelibrary.wiley.com/doi/pdf/10.1111/j.1467-8659.2011.01998.x

[89] Kai Rohmer, Wolfgang Büschel, Raimund Dachselt, and Grosch Thorsten. 2015. Interactive Near-Field Illumination for Photorealistic Augmented Reality with Varying Materials on Mobile Devices. IEEE Transactions on Visualization and Computer Graphics 21, 12 (Dec 2015), 1349-1362. https://doi.org/10.1109/TVCG.2015.2450717

[90] Kai Rohmer and Thorsten Grosch. 2015. Tiled Frustum Culling for Differential Rendering on Mobile Devices. In 2015 IEEE International Symposium on Mixed and Augmented Reality. 37-42. https://doi.org/10.1109/ISMAR.2015.13

[91] Kai Rohmer, Johannes Jendersie, and Thorsten Grosch. 2017. Natural Environment Illumination: Coherent Interactive Augmented Reality for Mobile and Non-Mobile Devices. IEEE Transactions on Visualization and Computer Graphics 23, 11 (Nov 2017), 2474-2484. https://doi.org/10.1109/ TVCG.2017.2734426

[92] Imari Sato, Yoichi Sato, and Katsushi Ikeuchi. 1999. Acquiring a radiance distribution to superimpose virtual objects onto a real scene. IEEE Transactions on Visualization and Computer Graphics 5, 1 (Jan 1999), 1-12. https://doi.org/10.1109/2945.764865

[93] I. Sato, Y. Sato, and K. Ikeuchi. 2003. Illumination from shadows. IEEE Transactions on Pattern Analysis and Machine Intelligence 25, 3 (March 2003), 290-300. https://doi.org/10.1109/TPAMI.2003.1182093

[94] Yoichi Sato, Mark D. Wheeler, and Katsushi Ikeuchi. 1997. Object Shape and Reflectance Modeling from Observation. In Proceedings of the 24th Annual Conference on Computer Graphics and Interactive Techniques (SIGGRAPH âĂŹ97). ACM Press/Addison-Wesley Publishing Co., USA, 379-387. https://doi.org/10.1145/258734.258885

[95] Dieter Schmalstieg and Tobias Höllerer. 2016. Augmented reality: principles and practice. Addison-Wesley Professional.

[96] Tobias Schwandt and Wolfgang Broll. 2016. A Single Camera Image Based Approach for Glossy Reflections in Mixed Reality Applications. In 2016 IEEE International Symposium on Mixed and Augmented Reality (ISMAR). 37-43. https://doi.org/10.1109/ISMAR.2016.12

[97] Weiqi Shi, Zeyu Wang, Metin Sezgin, Julie Dorsey, and Holly Rushmeier. 2017. Material Design in Augmented Reality with In-Situ Visual Feedback. In Proceedings of the Eurographics Symposium on Rendering: Experimental Ideas \& Implementations (EGSR '17). Eurographics Association, Goslar, DEU, 93-103. https://doi.org/10.2312/sre.20171198

Manuscript submitted to ACM 
[98] Wonyoung Son, Bodam Nam, Taehyub Kim, and Hyunki Hong. 2012. Using environment illumination information in mobile Augmented Reality. In 2012 IEEE International Conference on Consumer Electronics (ICCE). 588-589. https://doi.org/10.1109/ICCE.2012.6161985

[99] Kevin Soulier, Matías Nicolás Selzer, and Martín Leonardo Larrea. 2016. Real-Time Estimation of Illumination Direction for Augmented Reality with Low-Cost Sensors. In XXII Congreso Argentino de Ciencias de la Computación (CACIC 2016).

[100] Jürgen Stauder. 1999. Augmented reality with automatic illumination control incorporating ellipsoidal models. IEEE Transactions on Multimedia 1, 2 (June 1999), 136-143. https://doi.org/10.1109/6046.766735

[101] Natsuki Sugano, Hirokazu Kato, and Keihachiro Tachibana. 2003. The effects of shadow representation of virtual objects in augmented reality. In The Second IEEE and ACM International Symposium on Mixed and Augmented Reality, 2003. Proceedings. 76-83. https://doi.org/10.1109/ISMAR.2003. 1240690

[102] Peter Supan, Ines Stuppacher, and Michael Haller. 2006. Image Based Shadowing in Real-Time Augmented Reality. International fournal of Virtual Reality 5, 3 (Jan. 2006), 1-7. https://doi.org/10.20870/IJVR.2006.5.3.2692

[103] Norimichi Tsumura, Minh Nguyet Dang, Takao Makino, and Yoichi Miyake. 2003. Estimating the Directions to Light Sources Using Images of Eye for Reconstructing 3D Human Face. Color and Imaging Conference 2003, 1, 77-81. https://www.ingentaconnect.com/content/ist/cic/2003/ 00002003/00000001/art00014

[104] Jonas Unger, Stefan Gustavson, and Anders Ynnerman. 2007. Spatially varying image based lighting by light probe sequences. The Visual Computer 23, 7 (2007), 453-465. https://doi.org/10.1007/s00371-007-0127-6

[105] Jonas Unger, Joel Kronander, Per Larsson, Stefan Gustavson, Joakim Löw, and Anders Ynnerman. 2013. Spatially varying image based lighting using HDR-video. Computers \& graphics 37, 7 (2013), 923-934.

[106] Jonas Unger, Joel Kronander, Per Larsson, Stefan Gustavson, and Anders Ynnerman. 2013. Temporally and spatially varying image based lighting using HDR-video. In 21st European Signal Processing Conference (EUSIPCO 2013). 1-5.

[107] Henrique Weber, Donald Prévost, and Jean-François Lalonde. 2018. Learning to Estimate Indoor Lighting from 3D Objects. In 2018 International Conference on 3D Vision (3DV). 199-207. https://doi.org/10.1109/3DV.2018.00032

[108] Harald Wuest, Florent Vial, and Didier Stricker. 2005. Adaptive line tracking with multiple hypotheses for augmented reality. In Fourth IEEE and ACM International Symposium on Mixed and Augmented Reality (ISMAR'05). 62-69. https://doi.org/10.1109/ISMAR.2005.8

[109] Guanyu Xing, Yanli Liu, Xueying Qin, and Qunsheng Peng. 2011. On-line Illumination Estimation of Outdoor Scenes Based on Area Selection for Augmented Reality. In 2011 12th International Conference on Computer-Aided Design and Computer Graphics. 439-442. https://doi.org/10.1109/ CAD/Graphics.2011.51 